UC-702

Issued: October 1995

Spatial and Frequency Coherence of

Oblique, One-Hop, High-Frequency Paths

T. Joseph Fitzgerald 


\section{DISCLAIMER}

Portions of this document may be illegible in electronic image products. Images are produced from the best available original document. 


\title{
SPATIAL AND FREQUENCY COHERENCE OF OBLIQUE, ONE-HOP HIGH-FREQUENCY PATHS
}

by

\author{
T. Joseph Fitzgerald
}

\begin{abstract}
We consider the effect of random index of refraction fluctuations upon long-distance, ionospherically-reflected, hf paths. Along with deterministic effects such as multipath and dispersion, such fluctuations have a deleterious impact on hf communication including nonabsorptive fading, time-of-arrival spread, angle-of-arrival spread, and Doppler spread. We develop a formalism to calculate the mutual coherence functions for spatial and frequency separations based upon a path integral solution of the parabolic wave equation for a single refracted path through an ionosphere which contains random electron density fluctuations. The statistics of the hf path depend directly on the strength and statistics of the electron density fluctuations; we model the spatial power spectrum of the density fluctuation as a power law behavior versus frequency and with outer and inner scales.
\end{abstract}

\section{INTRODUCTION}

A perennial problem in the use of hf communication paths which reflect from the ionosphere has been the fact that the ionosphere is not a perfect mirror but, rather, behaves like a moving, distorted surface over a wide range of scale sizes. Such distortions arise from electron density fluctuations caused by a number of physical processes depending on location and time; the net result is that the hf communication path is deteriorated due to fading, multipath, pulse spreading, etc. To predict the statistics of hf paths which reflect from an ionosphere which contains random macroscopic fluctuations in electron density we employ a method developed to describe acoustic propagation in the oceanic sound channel [Flatté, 1983]. In this method the scalar wave equation is approximated for long distance propagation with only small deflections by the parabolic wave equation which can be cast in the form of Schrödinger's equation and thus has a path integral solution [Flatté, 1986]. The statistics of the received amplitude may be calculated from the formal path integral without calculating a realization of the solution. Although the interaction of electromagnetic waves with 
electrons is the problem of quantum electrodynamics and thus is amenable to a direct path integral solution [Feynman and Hibbs, 1965] we do not here consider such an approach to the hf propagation problem.

We develop a formalism to calculate the statistics of single-mode hf transmissions over oblique one-hop paths. The background electron density may have an arbitrary spatial dependence although our assumption of small angular deflection precludes steep gradients in the refractive index. The random spatial structure of the electron density is assumed to be characterized statistically by a power law spectrum with outer and inner scales. The spectral index, outer scale, inner scale, and the total variance may be functions of spatial location and altitude. The time variation of the random electron density is assumed to arise from the transport of fluctuations at a constant velocity and thus is controlled by the spatial statistics and the transport velocity.

The lowest order statistics that are of interest are the second moments of the wave amplitude for spatial, time, and frequency separations. These are called the mutual coherence functions and characterize the angular spread of the received transmissions (spatial separation), Doppler spread (time separation) and coherent bandwidth (frequency separation). Our results predict the ensemble average behavior of the mutual coherence functions. The interpretation of a given measurement must relate the time scale of the measurement to the time scale of ionospheric fluctuation. That is, one must determine whether the measurement is long enough to represent an ensemble average or whether it is only a single realization. These time scales depend on the type of measurement and upon ionospheric conditions. A typical example is Doppler spread over a mid-latitude path. A 100 second sample of data may show a narrow Doppler peak with a small offset. On the other hand, 10,000 seconds of data would show a broader Doppler peak centered at zero frequency. The broadening would arise from the averaging of random Doppler shifts over the longer time period [Flatté et al., 1979]. 


\section{PATH INTEGRAL SOLUTION OF THE PARABOLIC WAVE EQUATION}

We adopt a rectangular coordinate system in which the $\mathrm{x}$ axis connects the transmitter at $x=0$ with the receiver at $x=R$; the $\mathrm{z}$ axis is vertical and the $\mathrm{y}$ axis is orthogonal to both. We consider a wave of radian frequency $\omega$ and free space wave number $k_{o}$ so that $\omega=k_{o} c_{o}$, where $c_{o}$ is the speed of light in vacuum. We designate the phase speed of light in the ionosphere as $c$. Then the solution, $\Psi(\mathbf{r}, t)$, at position $\mathbf{r}$ and time $t$ of the scalar wave equation is approximately a plane wave propagating in the $\mathrm{x}$ direction. For the conditions outlined above, the reduced amplitude, $\psi(\mathbf{r}, t)$, given by

$$
\Psi(\mathbf{r}, t)=\psi(\mathbf{r}, t) e^{i\left(k_{o} x-\omega t\right)}
$$

satisfies a parabolic wave equation

$$
-\frac{1}{2 k_{o}^{2}}\left(\frac{\partial^{2} \psi}{\partial z^{2}}+\frac{\partial^{2} \psi}{\partial y^{2}}\right)+U \psi=\frac{i}{k_{o}} \frac{\partial \psi}{\partial x},
$$

where we have used $U=\frac{1}{2}\left(1-\frac{c_{0}^{2}}{c^{2}}\right)$. Equation (2) has been cast in a form of Schrödinger's equation

$$
-\frac{\hbar^{2}}{2 m} \nabla^{2} \psi+V \psi=i \hbar \frac{\partial \psi}{\partial t}
$$

The horizontal distance, $x$, in Eq. (2) is the analog of time in Schrödinger's equation. The path integral solution of Schrödinger's equation is

$$
\psi=\int_{a}^{b} D[\mathbf{x}(t)] e^{(i / \hbar) S[b, a]}
$$

for a particle moving from point $a$ at time $t_{a}$ to point $b$ at time $t_{b}$. The factor $D[\mathbf{x}(t)]$ represents a sum over all possible paths; $S[b, a]$ is the action over a given path, $x(t)$, and is equal to

$$
S=\int_{t_{a}}^{t_{b}}\left[\frac{m}{2} \dot{\mathbf{x}}^{2}-V(\mathbf{x}, t)\right] d t
$$

By analogy $\left(m=1, \hbar=\frac{1}{k_{o}}\right)$ the path integral solution of Eq. (2) is

$$
\psi=\int_{0}^{R} D[y(x), z(x)] \exp \left(i k_{0} S[y(x), z(x)]\right),
$$


where

$$
S[y(x), z(x)]=\int_{0}^{R}\left\{\frac{1}{2}\left[\left(\frac{\partial y}{\partial x}\right)^{2}+\left(\frac{\partial z}{\partial x}\right)^{2}\right]-U\right\} d x .
$$

The amplitude given by Eq. (6) implicitly assumes that the antenna responses are spatially isotropic. Otherwise, each path would have to be weighted according to direction.

We will write the path integral solution for $\psi$, Eq. (6), as

$$
\psi=\mathcal{N} \int D \mathbf{z} \exp \left(i k_{o} S_{o}(\mathbf{z})-i k_{o} \int_{o}^{R} \mu[x, \mathbf{z}(x), t] d x\right)
$$

where $\mathcal{N}$ is a normalization factor and $k_{0} S_{o}(\mathbf{z})$ is the phase associated with path $\mathbf{z}(x)=[y(x), z(x)]$ in the absence of fluctuations:

$$
k_{o} S_{o}(\mathbf{z})=k_{o} \int_{o}^{R}\left[\frac{1}{2}\left(\frac{\partial y}{\partial x}\right)^{2}+\frac{1}{2}\left(\frac{\partial z}{\partial x}\right)^{2}-U_{o}(\mathbf{z})\right] d x
$$

We use the following equation for $U$ :

$$
U=U_{o}(\mathbf{z})+\mu(\mathbf{z}, t)
$$

where $U_{o}$ represents the deterministic ionospheric profile and $\mu$ is a random, zero-mean function incorporating the effects of the fluctuations. We assume that $U_{o}$ is only a function of height, $U_{o}=U_{o}(z)$. Since $\frac{c_{o}}{c}$ is the index of refraction, $U_{o}=\frac{1}{2}\left(1-n_{o}^{2}\right)$, where $n_{o}(z)$ is the deterministic index of refraction and $\mu \simeq-n_{o} \Delta n$, where $\Delta n(\mathbf{r}, t)$ represents the random component of $n$. For oblique propagation $n_{o} \simeq 1$ and $\mu \simeq-\Delta n$. Since $U$ is small, the phase speed is approximately

$$
c=c_{o}\left[1+U_{o}(z)+\mu(\mathbf{r}, t)\right]
$$

\section{ELECTRON DENSITY FLUCTUATIONS}

The index of refraction fluctuations, $\mu(\mathbf{r}, t)$, are produced by random electron density variations in the ionosphere. These density variations arise naturally in the ionosphere from waves in the neutral atmosphere or by structuring of the ionospheric plasma. They will usually also be present 
in any artificial modification of the ionosphere. Assuming that the ratio of the random density variations, $\Delta N(\mathbf{r}, t)$, to the deterministic electron density, $N_{o}(z)$, is sufficiently small we may write using Eq. (10)

$$
\mu(\mathbf{r}, t)=\frac{\partial U_{o}}{\partial N} \Delta N(\mathbf{r}, t)
$$

The fluctuation, $\mu$, will enter our results only through its covariance

$$
\left\langle\mu\left(\mathbf{r}^{\prime}, t^{\prime}\right) \mu(\mathbf{r}, t)\right\rangle=\left\langle\frac{\partial U_{o}\left(\mathbf{r}^{\prime}\right)}{\partial N} \Delta N\left(\mathbf{r}^{\prime}, t^{\prime}\right) \frac{\partial U_{o}(\mathbf{r})}{\partial N} \Delta N(\mathbf{r}, t)\right\rangle
$$

We assume that the correlation distances of the electron density fluctuations, $\Delta N$, are sufficiently small so that

$$
\frac{\partial U_{o}\left(\mathbf{r}^{\prime}\right)}{\partial N} \simeq \frac{\partial U_{o}(\mathbf{r})}{\partial N}
$$

and that for the deterministic electron density

$$
N_{o}\left(\mathbf{r}^{\prime}\right) \simeq N_{o}(\mathbf{r})
$$

Then

$$
\left\langle\mu\left(\mathbf{r}^{\prime}, t^{\prime}\right) \mu(\mathbf{r}, t)\right\rangle=\left(N_{\circ} \frac{\partial U_{0}}{\partial N}\right)^{2} \rho_{N}\left(\mathbf{r}^{\prime}, t^{\prime}, \mathbf{r}, t\right),
$$

where $\rho_{N}$ is the covariance of the relative electron density variations and

$$
\rho_{N}\left(\mathbf{r}^{\prime}, t^{\prime}, \mathbf{r}, t\right)=\frac{\left\langle\Delta N\left(\mathbf{r}^{\prime}, t^{\prime}\right) \Delta N(\mathbf{r}, t)\right\rangle}{N_{o}\left(\mathbf{r}^{\prime}\right) N_{o}(\mathbf{r})}
$$

We assume that $\left\langle(\Delta N)^{2}\right\rangle$ changes sufficiently slowly over the spatial and temporal correlation scales that we may write $\rho_{N}$ as the product of the variance of $\Delta N / N_{0}$ and the (stationary) autocorrelation function, $C$. That is,

$$
\rho_{N}\left(\mathbf{r}^{\prime}, t^{\prime}, \mathbf{r}, t\right)=\left\langle\left(\frac{\Delta N(\mathbf{r}, t)}{N_{o}(\mathbf{r})}\right)^{2}\right\rangle C\left(\mathbf{r}^{\prime}-\mathbf{r}, t^{\prime}-t\right)
$$

so that

$$
\left\langle\mu\left(\mathbf{r}^{\prime}, t^{\prime}\right) \mu(\mathbf{r}, t)\right\rangle=\left\langle\mu^{2}(\mathbf{r}, t)\right\rangle C\left(\mathbf{r}^{\prime}-\mathbf{r}, t^{\prime}-t\right)
$$


where

$$
\left\langle\mu^{2}(\mathbf{r}, t)\right\rangle=\left(N_{o}(\mathbf{r}) \frac{\partial U_{o}(\mathbf{r})}{\partial N}\right)^{2}\left\langle\left(\frac{\Delta N(\mathbf{r}, t)}{N_{o}(\mathbf{r})}\right)^{2}\right\rangle .
$$

In the high frequency limit, $\omega^{2}>\omega_{0}^{2}$, where

$$
\omega_{o}^{2}=\frac{N_{o} e^{2}}{m_{e} \epsilon_{o}}
$$

$e$ is the electron charge, $m_{e}$ the electron mass, and $\epsilon_{o}$ the permittivity of free space, $U_{o}$ is approximately

$$
U_{o} \simeq \frac{1}{2} \frac{\omega_{0}^{2}}{\omega^{2}}
$$

and

$$
N_{o} \frac{\partial U_{o}}{\partial N} \simeq \frac{1}{2} \frac{\omega_{o}^{2}}{\omega^{2}}
$$

The dependence of $\rho_{N}$ on $\Delta \mathbf{r}=\mathbf{r}^{\prime}-\mathbf{r}$ is subject to debate both for the natural ionosphere and for man-made disturbances. However, it is clear that in the natural ionosphere the variances of $\rho_{N}$ and $\left\langle(\Delta N / N)^{2}\right\rangle$ show a strong dependence on latitude as well as time of day, season, sunspot number, altitude, etc. The dependence of $\rho_{N}$ on $\Delta t=t^{\prime}-t$ is also uncertain and may depend on the physical process generating the fluctuations. The frozen transport assumption is commonly made, that is, time dependence is generated by the motion of spatial structures at some deterministic velocity $\mathbf{v}$ so that

$$
\rho_{N}(0, \Delta t)=\rho_{N}(\mathbf{v} \Delta t, 0)
$$

The spatial scale of the transport velocity is assumed to be much larger than the scale of the density fluctuations. On the other hand, another simple model would be to assume that the time dependence is controlled by some decorrelation time, $T$, so that

$$
\rho_{N}(0, \Delta t)=\rho_{N}(0,0) e^{-\frac{1}{2}\left(\frac{\Delta t}{T}\right)^{2}}
$$

and is separable from the spatial dependence.

Figure 1 shows examples of power spectra of relative electron density fluctuations, $\Delta N / N_{o}$, versus spatial wavenumber, $\kappa$, measured along rocket trajectories through a barium ion cloud and 
through equatorial spread F. The processes thought to cause the electron density fluctuations have related physics and the similar power law dependence of the spectra supports the theory. These and similar measurements support a spectral index, SI, of between 1.5 and 2 , where $V(\kappa) \propto 1 / \kappa^{S I}$ in the power law regime.

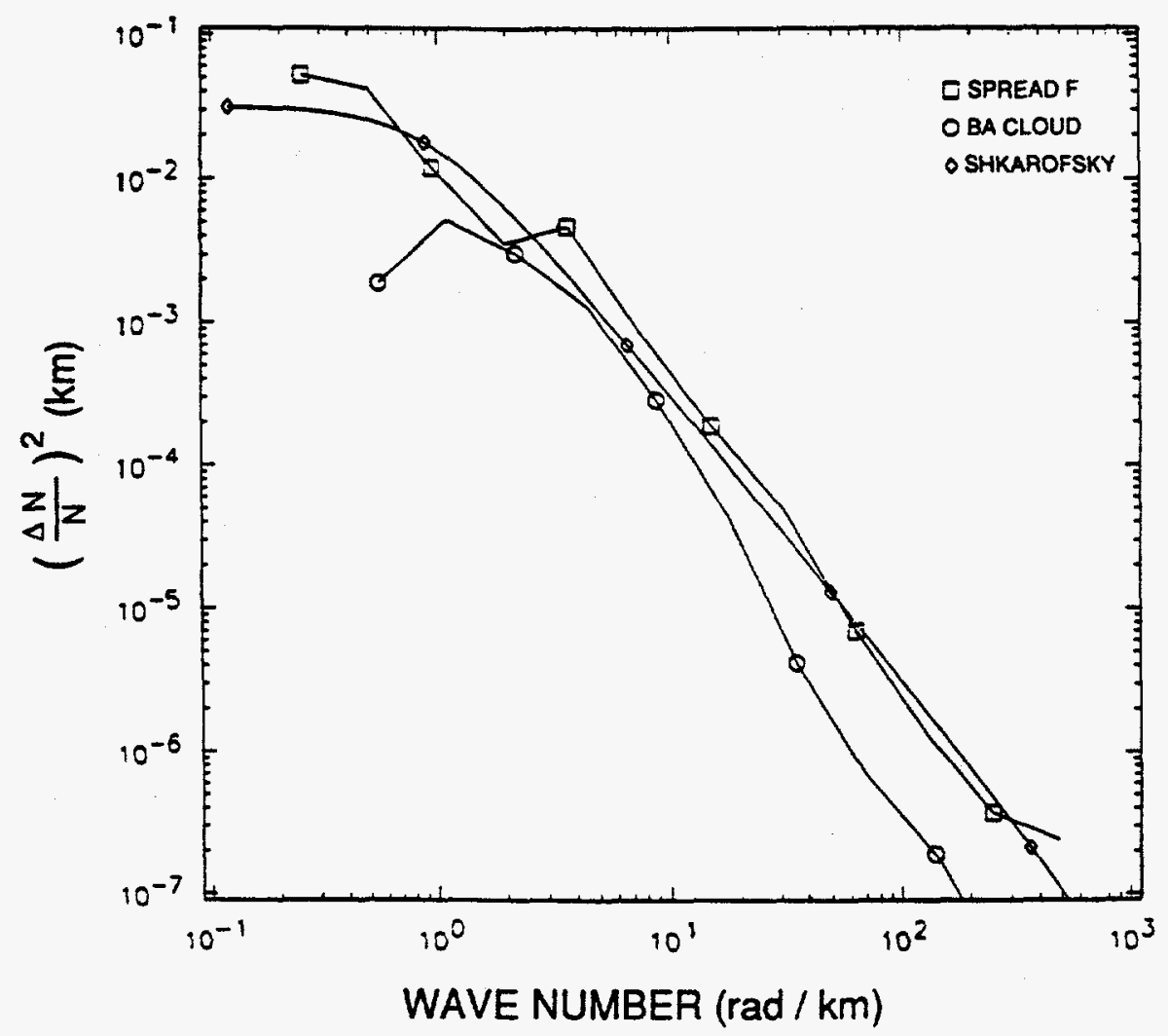

Fig. 1. Comparison of measured power spectra of electron density fluctuations in barium cloud and equatorial spread $\mathrm{F}$ with analytical spectrum [after Kelly et al., 1979].

It is convenient to employ an analytical form for the spatial autocorrelation function $C(\Delta r, 0)$. For the isotropic case, the spatial power spectrum may be characterized by three parameters: the spectral power-law decay index, $\nu$, the turnover wave number, $\kappa_{o}$, at which the power law decay begins (the inverse of the outer scale), and the turnover point at high frequency at which the power 
law changes to an exponential decay, $r_{0}$ (the inner scale). Shkarofsky [1968] proposed the following autocorrelation function, $C(r)$ :

$$
C(r)=\frac{\left(\kappa_{o} \sqrt{r^{2}+r_{o}^{2}}\right)^{\frac{\nu-1}{2}} K_{\frac{\nu-1}{2}}\left[\kappa_{o} \sqrt{r^{2}+r_{o}^{2}}\right]}{\left(\kappa_{o} r_{o}\right)^{\frac{\nu-1}{2}} K_{\frac{\nu-1}{2}}\left[\kappa_{o} r_{o}\right]},
$$

where $K$ is the modified Bessel function. The normalized one-dimensional spectrum, $V$, is given by

$$
V(\kappa)=\frac{1}{\sqrt{2 \pi}}\left(\frac{r_{o}}{\kappa_{o}}\right)^{1 / 2}\left(\frac{\kappa_{o}}{\sqrt{\kappa^{2}+\kappa_{o}^{2}}}\right)^{\nu / 2} \frac{K_{\nu / 2}\left[r_{o} \sqrt{\kappa^{2}+\kappa_{o}^{2}}\right]}{K_{\frac{\nu-1}{2}}\left[\kappa_{o} r_{o}\right]},
$$

where $K$ is the modified Bessel function. Figure 1 compares a Shkarofsky spectrum $V(\kappa)-$ with spectral index $\nu=2$; outer scale $\frac{1}{\kappa_{0}}=2.5 \mathrm{~km}$; and inner scale $r_{o}=15 \mathrm{~m}$ - with the measured spread $\mathrm{F}$ and $\mathrm{Ba}$ cloud spectra.

Shkarofsky [1968] generalized the autocorrelation function, $C(r)$, to include anisotropy. For example, if the electron density structures are field-aligned, but isotropic perpendicular to the field, we can write the autocorrelation function $C\left(r_{1}, r_{2}, r_{3}\right)$ as a function of three orthogonal coordinates, where $r_{1}$ is parallel to the field:

$$
C\left(r_{1}, r_{2}, r_{3}\right)=\frac{\left(\sqrt{\kappa_{o}^{2}\left(r_{o}^{2}+r_{2}^{2}+r_{3}^{2}\right)+w^{2} \kappa_{o}^{2} r_{1}^{2}}\right)^{\frac{\nu-1}{2}} K_{\frac{\nu-1}{2}}\left(\kappa_{o} \sqrt{r_{o}^{2}+r_{2}^{2}+r_{3}^{2}+w^{2} r_{1}^{2}}\right)}{\left(\kappa_{o} r_{o}\right)^{\frac{\nu-1}{2}} K_{\frac{\nu-1}{2}}\left(\kappa_{o} r_{o}\right)} .
$$

The parameter, $w$, is the ratio of the correlation length perpendicular to the field to the correlation length parallel to the field. For structures aligned along axis $1, w<1$. Although we associate the preferred axis with the magnetic field, the formalism is completely general.

We need to relate the axes used to define the correlation function $\left(r_{1}, r_{2}, r_{3}\right)$ to the ray trace coordinates $(x, y, z)$. Define $\theta$ as the elevation angle of the ray with respect to the horizontal, $\alpha$ as the azimuth of the ray with respect to magnetic north, and $\delta$ as the dip angle of the field (Figure 2). We label the Cartesian coordinates of the axes relative to the ray as $(\xi, \eta, \zeta)$ where $\xi$ is along the ray, $\zeta$ is normal downwards from the ray, and $\eta$ is horizontal perpendicular to $\xi$. The Cartesian 


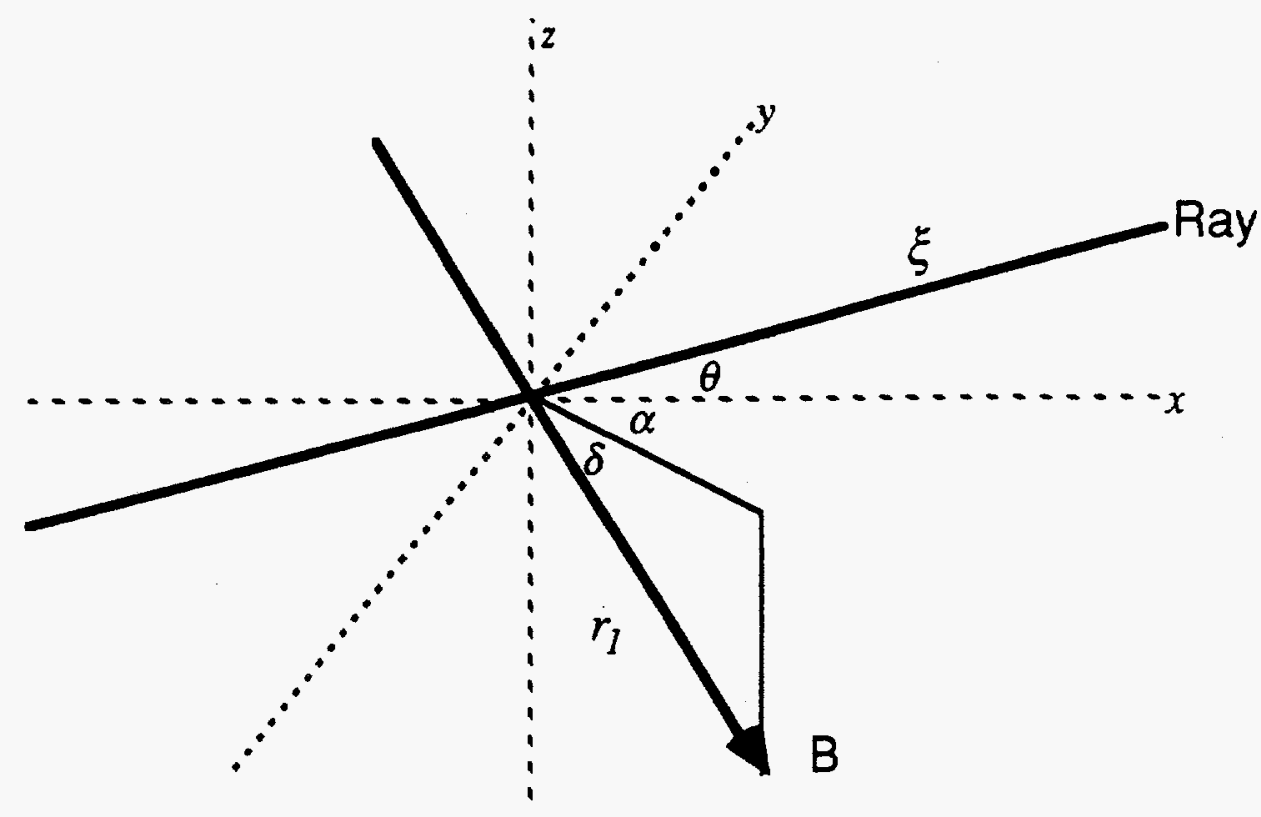

Fig. 2. Representation of coordinate systems: $(x, y, z)$ are rectangular coordinates used for ray tracing. The nominal ray path is in the $x-z$ plane at an angle $\theta$ to $x$ axis; $\xi$ is distance along ray. The preferred orientation of electron density fluctuations is along $B$ which has an azimuth $\alpha$ relative to $\mathrm{x}$ axis and dip angle $\delta ; r_{1}$ is distance along $\mathrm{B}$.

coordinates relative to the magnetic field system are $r_{1}$ along the field, $r_{2}$ horizontal east, and $r_{3}$ perpendicular to $r_{1}$ and $r_{2}$. The coordinate transformation is then

$$
\begin{aligned}
& r_{1}=\xi(\cos \delta \cos \alpha \cos \theta-\sin \delta \sin \theta)-\eta \cos \delta \sin \alpha+\zeta(\cos \delta \cos \alpha \sin \theta+\sin \delta \cos \theta) \\
& r_{2}=\xi \sin \alpha \cos \theta+\eta \cos \alpha+\zeta \sin \alpha \sin \theta \\
& r_{3}=-\xi(\sin \delta \cos \alpha \cos \theta+\cos \delta \sin \theta)+\eta \sin \delta \sin \alpha-\zeta(\sin \delta \cos \alpha \cos \theta-\cos \delta \cos \theta)
\end{aligned}
$$

Since

$$
r_{1}^{2}+r_{2}^{2}+r_{3}^{2}=\xi^{2}+\eta^{2}+\zeta^{2}
$$

we can write

$$
r_{o}^{2}+r_{2}^{2}+r_{3}^{2}+w^{2} r_{1}^{2}=r_{o}^{2}+\eta^{2}+\zeta^{2}+r_{1}^{2}\left(w^{2}-1\right)+\xi^{2}
$$

Write

$$
r_{1}=a \xi+b \eta+c \zeta
$$


where

$$
\begin{aligned}
& a=\cos \delta \cos \alpha \cos \theta-\sin \delta \sin \theta \\
& b=-\cos \delta \sin \alpha \\
& c=\cos \delta \cos \alpha \sin \theta+\sin \delta \cos \theta
\end{aligned}
$$

then

$$
r_{o}^{2}+r_{2}^{2}+r_{3}^{2}+w^{2} r_{1}^{2}=r_{o}^{2}+\eta^{2}+\zeta^{2}+\left(w^{2}-1\right)\left[a^{2} \xi^{2}+2 a \xi(b \eta+c \zeta)+(b \eta+c \zeta)^{2}+\frac{\xi^{2}}{w^{2}-1}\right]
$$

which can be rearranged as

$$
r_{o}^{2}+r_{2}^{2}+r_{3}^{2}+w^{2} r_{1}^{2}=r_{o}^{2}+\eta^{2}+\zeta^{2}+\frac{(b \eta+c \zeta)^{2}\left(w^{2}-1\right)}{a^{2}\left(w^{2}-1\right)+1}+\left[a^{2}\left(w^{2}-1\right)+1\right]\left[\xi+\frac{a(b \eta+c \zeta)\left(w^{2}-1\right)}{a^{2}\left(w^{2}-1\right)+1}\right]^{2} \text {. }
$$

\subsection{Phase fluctuations}

The phase, $\phi$, of the wave is given by

$$
\phi=\int k d s
$$

where $k$ is the radian wave number and $d s$ is an element of the ray path. The wave number can be written as $n k_{o}$ where $k_{o}$ is the free space wave number and $n$ is the index of refraction. Call $\phi_{o}$ the phase in the absence of fluctuations so that the phase fluctuation, $\Delta \phi$, is

$$
\Delta \phi=\phi-\phi_{o}=k_{o} \int\left(n-n_{o}\right) d s
$$

or

$$
\Delta \phi=k_{o} \int \frac{\partial n}{\partial N} \Delta N d s
$$

But $\frac{\partial n}{\partial N} \simeq-\frac{\partial U}{\partial N}$ so, using Eq. (12), we find

$$
\Delta \phi=-k_{o} \int \mu d s
$$


The variance of the phase fluctuations, $\Phi^{2}$, is an important parameter in characterizing the strength of the disturbance caused by the random structure. It can be expressed as

$$
\Phi^{2}=\left\langle(\Delta \phi)^{2}\right\rangle=k_{o}^{2}\left\langle\left(\int d s \mu(s)\right)^{2}\right\rangle
$$

or

$$
\Phi^{2}=k_{o}^{2} \int d s \int d s^{\prime}\left\langle\mu(s) \mu\left(s^{\prime}\right)\right\rangle
$$

Using Eq. (19) we can write

$$
\Phi^{2}=k_{o}^{2} \int d s\left\langle\mu^{2}(s)\right\rangle \int d s^{\prime} C\left(s-s^{\prime}\right)
$$

where $s-s^{\prime}$ represents a separation along the same ray path. Defining the correlation length, $L_{p}$, tangent to the ray path as

$$
L_{p}(s, \theta, \alpha)=\int_{-\infty}^{\infty} d s^{\prime} C\left(s-s^{\prime}\right)
$$

we can write

$$
\Phi^{2}=k_{o}^{2} \int d s\left\langle\mu^{2}(s)\right\rangle L_{p}(s, \theta, \alpha)
$$

assuming that $C\left(s-s^{\prime}\right)$ falls off sufficiently fast that the ray does not change orientation significantly. The parallel correlation length may depend on the orientation of the ray through the elevation angle, $\theta$, and the azimuth, $\alpha$ [Flatté et al., 1979].

\subsection{Parallel correlation length}

Since $\xi=s^{\prime}-s$, the parallel correlation length, $L_{p}(s, \theta, \alpha)$, defined in Eq. (47), is given by

$$
L_{p}(s, \theta, \alpha)=\int_{-\infty}^{\infty} d \xi C(\xi, 0,0)
$$

Substituting the coordinate transformation given by Eq. (39) with $\eta=\zeta=0$ into the autocorrelation function, Eq. (28), we obtain

$$
L_{p}(s, \theta, \alpha)=\int_{-\infty}^{\infty} d \xi \frac{\left(\sqrt{\kappa_{o}^{2} r_{o}^{2}+A \kappa_{o}^{2} \xi^{2}}\right)^{\frac{\nu-1}{2}}}{\left(\kappa_{o} r_{o}\right)^{\frac{\nu-1}{2}} K_{\frac{\nu-1}{2}}\left(\kappa_{o} r_{o}\right)} K_{\frac{\nu-1}{2}}\left(\kappa_{o} \sqrt{r_{o}^{2}+A \xi^{2}}\right)
$$


where

$$
A=1+\left(w^{2}-1\right) a^{2}
$$

Then, changing variables,

$$
L_{p}(s, \theta, \alpha)=\frac{2}{\sqrt{A}\left(\kappa_{o} r_{o}\right)^{\frac{\nu-1}{2}}} \frac{\kappa_{o}^{\frac{\nu-1}{2}}}{K_{\frac{\nu-1}{2}}\left(\kappa_{o} r_{o}\right)} \int_{o}^{\infty} d x\left(\sqrt{r_{o}^{2}+x^{2}}\right)^{\frac{\nu-1}{2}} K_{\frac{\nu-1}{2}}\left(\kappa_{o} \sqrt{r_{o}^{2}+x^{2}}\right),
$$

which gives

$$
L_{p}(s, \theta, \alpha)=\sqrt{\frac{2 \pi}{A}}\left(\frac{r_{o}}{\kappa_{o}}\right)^{1 / 2} \frac{K_{\frac{\nu}{2}}\left(\kappa_{o} r_{o}\right)}{K_{\frac{\nu-1}{2}}\left(\kappa_{o} r_{o}\right)}
$$

Since $\kappa_{o} r_{o} \ll 1$

$$
K_{\frac{\nu}{2}}\left(\kappa_{o} r_{o}\right) \simeq \frac{1}{2} \Gamma\left(\frac{\nu}{2}\right)\left(\kappa_{o} r_{o}\right)^{\frac{-\nu}{2}}
$$

so that

$$
L_{p}(s, \theta, \alpha) \simeq \frac{1}{\kappa_{o}} \sqrt{\frac{2 \pi}{A}} \frac{\Gamma\left(\frac{\nu}{2}\right)}{\Gamma\left(\frac{\nu-1}{2}\right)}
$$

to a high degree of accuracy. The correlation length may depend on position along the ray, $s$, since the parameters $r_{o}, \kappa_{o}$, and $w$ need not be constant. With this analytical result for $L_{p}, \mathrm{Eq}$. (48) can be integrated numerically along the ray to obtain $\Phi^{2}$. The parallel correlation length shows a strong dependence on spectral index, $\nu$, as can be seen from Figure 3, where we plot $\sqrt{A} \kappa_{o} L_{p}$ versus $\nu$. The higher the spectral index, the more the low spatial frequencies contribute to the fluctuations and thus the greater the correlation length. Since $\Phi^{2}$ is proportional to $L_{p}$, for the same electron density variance, the phase variance will increase with increasing spectral index.

\section{SAME FREQUENCY MUTUAL COHERENCE FUNCTION}

The mutual coherence function (mcf), defined as $\left\langle\psi^{*}(2) \psi(1)\right\rangle$, where $\psi(2)$ is the wave amplitude at receiver $2, \psi(1)$ at receiver 1 , and 1 and 2 indicate space and time separation, has been derived from the path integral solution for $\psi$ for oblique propagation at a single transmission frequency [Dashen, Flatté, and Reynolds, 1985]. If the receiver is not located at a caustic, the mcf can be written in terms of the phase structure function, $\mathrm{D}(1,2)$,

$$
\left\langle\psi^{*}(2) \psi(1)\right\rangle=\exp \left[-\frac{1}{2} D(1,2)\right]
$$




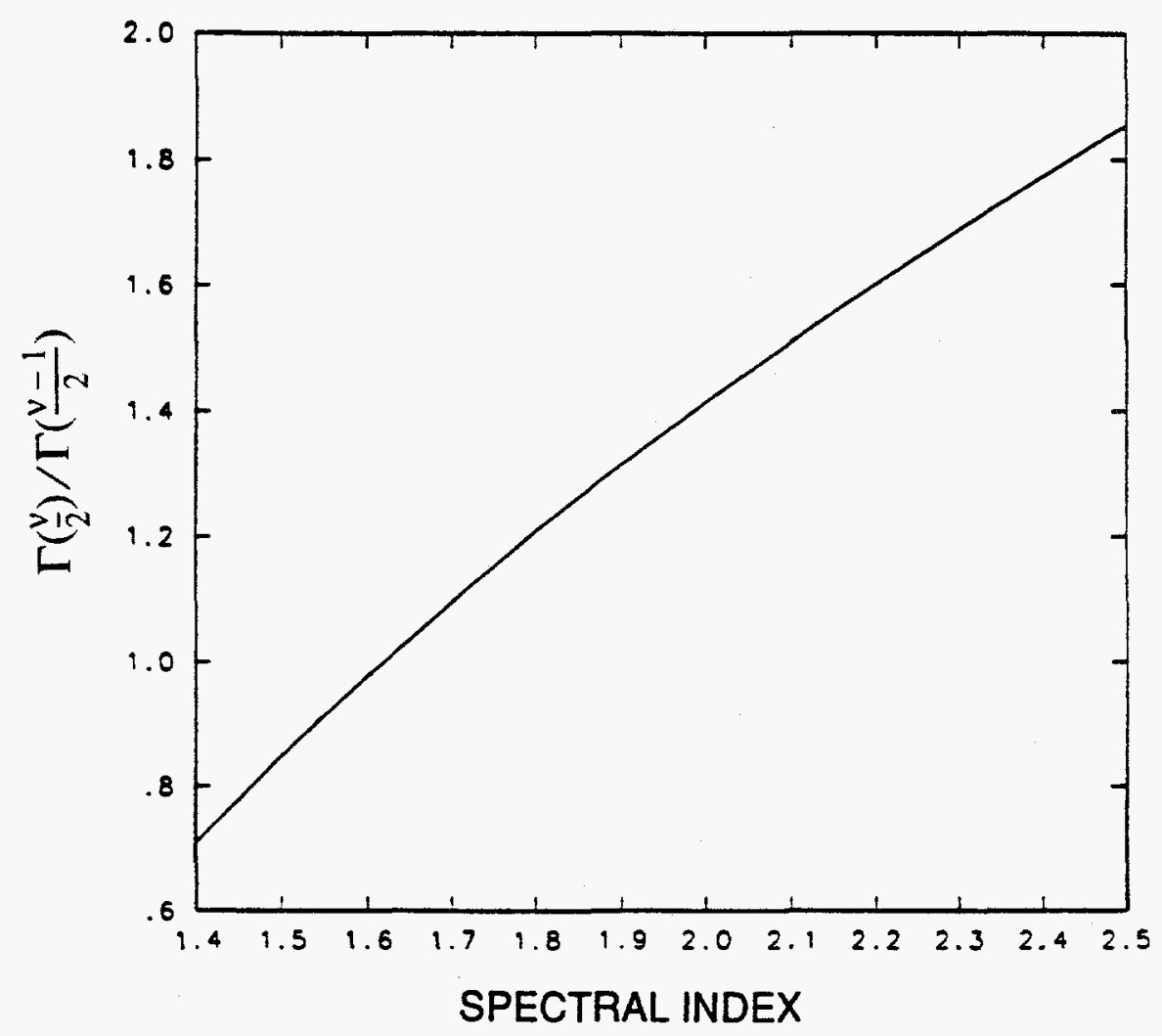

Fig. 3. Plot of $\Gamma\left(\frac{\nu}{2}\right) / \Gamma\left(\frac{\nu-1}{2}\right)$ versus spectral index $\nu$ showing from Eq. (55) that the parallel correlation length, $L_{p}$, increases with $\nu$.

where $D(1,2)$ is defined [Esswein and Flatté, 1981] as

$$
D(1,2) \equiv k_{o}^{2}\left\langle\left(\int_{1} \mu d s-\int_{2} \mu d s\right)^{2}\right\rangle
$$

where the integrals are taken along the deterministic paths to the two receivers. In terms of the covariance, $\rho$, of the index of refraction variations, where

$$
\rho\left(\mathbf{x}, t, \mathbf{x}^{\prime}, t^{\prime}\right)=\left\langle\mu(\mathbf{x}, t) \mu\left(\mathbf{x}^{\prime}, t^{\prime}\right)\right\rangle
$$

$D(1,2)$ may be written as

$$
D(1,2)=\int_{1} d s\left\{2 k_{o}^{2}\left(\int_{1} d s^{\prime} \rho\left(s, s^{\prime}\right)-\int_{2} d s^{\prime} \rho\left(s, s^{\prime}\right)\right)\right\}
$$


[Esswein and Flatté, 1981]. If the ray curvature is insignificant over the correlation length of the fluctuations, the rays may be approximated by the tangents to the ray path at $s$. Furthermore, assuming that the rays 1 and 2 are close, ray 2 will be parallel to ray 1 . The first integral in parenthesis in Eq. (59) becomes by the definition of $L_{p}$

$$
\int_{1} d s^{\prime} \rho\left(s, s^{\prime}\right)=\left\langle\mu^{2}(s)\right\rangle L_{p}(\theta, s)
$$

The second integral is a function of the separation between the rays, $\Delta$, so that $\mathrm{D}(1,2)$ may be written as

$$
D(1,2)=2 k_{o}^{2} \int d s \int d s^{\prime}\left[\rho\left(s, s^{\prime}\right)-\rho\left(s, s^{\prime}+\Delta\right)\right]
$$

or

$$
D(1,2)=2 k_{o}^{2} \int d s\left\langle\mu^{2}(s)\right\rangle L_{p}(\theta, s) f(\Delta ; \theta, s)
$$

where $f(\Delta ; \theta, s)$ is called the phase correlation function [Esswein and Flatté, 1981]. The phase correlation function can be written as

$$
f(\Delta ; \theta, s)=1-\frac{\int d s^{\prime} \rho\left(s, s^{\prime}+\Delta\right)}{\left\langle\mu^{2}(s)\right\rangle L_{p}(\theta, s)}
$$

or using Eq. (19)

$$
f(\Delta ; \theta, s)=1-\frac{1}{L_{p}(\theta, s)} \int d s^{\prime} C\left(s, s^{\prime}+\Delta\right)
$$

Consider rays separated by $\Delta z$ in the vertical direction; they are separated by $\zeta=\Delta z \cos \theta$ using the coordinates relative to the ray. Then

$$
\int d s^{\prime} C\left(s, s^{\prime}+\Delta\right)=\int d \xi C(\xi, 0, \Delta z \cos \theta)
$$

Changing variables in the form given by Eq. (39) we obtain

$$
\int d s^{\prime} C\left(s, s^{\prime}+\Delta\right)=\frac{1}{\sqrt{A}} \int d v C\left(r_{o}^{2}+B(\cos \theta \Delta z)^{2}+v^{2}\right)
$$

where

$$
B=1+\frac{c^{2}\left(w^{2}-1\right)}{A}
$$


The integral gives

$$
\begin{aligned}
\int d s^{\prime} C\left(s, s^{\prime}+\Delta\right) & =\frac{\sqrt{2 \pi}}{\sqrt{A}}\left(\frac{r_{o}}{\kappa_{o}}\right)^{1 / 2} \\
& \times\left(\sqrt{1+B\left(\frac{\cos \theta \Delta z}{r_{o}}\right)^{2}}\right)^{\frac{\nu}{2}} \frac{K_{\frac{\nu}{2}}\left(\kappa_{o} \sqrt{r_{o}^{2}+B(\cos \theta \Delta z)^{2}}\right)}{K_{\frac{\nu-1}{2}}\left(\kappa_{o} r_{o}\right)}
\end{aligned}
$$

The phase correlation function for vertical separation, $f(\Delta z ; \theta, s)$, is then

$$
f(\Delta z ; \theta, s)=1-\left(\sqrt{1+B\left(\frac{\cos \theta \Delta z}{r_{o}}\right)^{2}}\right)^{\frac{\nu}{2}} \frac{K_{\frac{\nu}{2}}\left(\kappa_{o} \sqrt{r_{o}^{2}+B(\cos \theta \Delta z)^{2}}\right)}{K_{\frac{\nu}{2}}\left(\kappa_{o} r_{o}\right)} .
$$

Now consider rays separated by $\Delta y$ in the horizontal direction and again assume that the rays are parallel. The integral corresponding to Eq. (65) is

$$
\int d s^{\prime} C\left(s, s^{\prime}+\Delta\right)=\int d \xi C(\xi, \Delta y, 0)
$$

Again changing variables we find

$$
\int d s^{\prime} C\left(s, s^{\prime}+\Delta y\right)=\frac{1}{\sqrt{A}} \int d v C\left(r_{o}^{2}+D(\Delta y)^{2}+v^{2}\right)
$$

where

$$
D=1+\frac{b^{2}\left(w^{2}-1\right)}{A}
$$

By analogy with Eq. (68)

$$
\int d s^{\prime} C\left(s, s^{\prime}+\Delta y\right)=\frac{\sqrt{2 \pi}}{\sqrt{A}}\left(\frac{r_{o}}{\kappa_{o}}\right)^{1 / 2}\left(\sqrt{1+D\left(\frac{\Delta y}{r_{o}}\right)^{2}}\right)^{\frac{\nu}{2}} \frac{K_{\frac{\nu}{2}}\left(\kappa_{o} \sqrt{r_{o}^{2}+D(\Delta y)^{2}}\right)}{K_{\frac{\nu-1}{2}}\left(\kappa_{o} r_{o}\right)} .
$$

The phase correlation function for horizontal separation, $f(\Delta y ; \theta, s)$, is then

$$
f(\Delta y ; \theta, s)=1-\left(\sqrt{1+D\left(\frac{\Delta y}{r_{0}}\right)^{2}}\right)^{\frac{\nu}{2}} \frac{K_{\frac{\nu}{2}}\left(\kappa_{o} \sqrt{r_{o}^{2}+D(\Delta y)^{2}}\right)}{K_{\frac{\nu}{2}}\left(\kappa_{o} r_{0}\right)} .
$$

Given the phase correlation functions one can numerically integrate Eq. (62) for $D(1,2)$ for ray separations in the vertical or horizontal direction. In a horizontally stratified ionosphere, ray 
separations in the vertical direction arise from differences in take off angle while separations in the horizontal direction arise from differences in azimuth. Given receivers with horizontal separation, $\Delta$, normal to the ray path at a range $R$ from the transmitter, the separation, $\Delta y$, between rays at a distance $x$ from the transmitter is

$$
\Delta y=\frac{x}{R} \Delta
$$

On the other hand, receivers separated by a distance $\Delta$ parallel to the ray may be thought of as having a vertical separation $\tilde{\Delta}$ at range $R$, where $\tilde{\Delta}=\Delta \tan \theta_{0}$ and $\theta_{o}$ is the elevation angle of the ray at the receiver. That is, we are assuming the receivers are near the ground below the ionosphere so that there is no contribution to $D(1,2)$ between the equivalent vertical receivers and the actual horizontal receivers. Moreover, from Snell's law, $\theta_{0}$ is also the elevation angle of the rays at the transmitter which we assume is also located near the ground. However, the vertical separation $\Delta z(x)$ between rays does not have as simple a relation to $\tilde{\Delta}$ as that given by Eq. (75). One could determine $\Delta z$ by ray tracing to different receiver separations $\Delta$, but it is possible to reduce the computational effort by using the concept of a ray tube which is defined in terms of a linear differential equation for the displacement of a ray from the nominal path [Flatté et al., 1979].

Returning to the phase correlation function for the most general case of a displacement $\Delta y$ in the horizontal direction and $\Delta z$ in the vertical direction we find that

$$
\begin{aligned}
f(\Delta ; \theta, s)=1- & \left(\sqrt{1+\frac{B(\cos \theta \Delta z)^{2}+2 E \cos \theta \Delta z \Delta y+D(\Delta y)^{2}}{r_{o}^{2}}}\right)^{\frac{\nu}{2}} \\
& \times \frac{K_{\frac{\nu}{2}}\left(\kappa_{o} \sqrt{r_{o}^{2}+B(\cos \theta \Delta z)^{2}+2 E \cos \theta \Delta z \Delta y+D(\Delta y)^{2}}\right)}{K_{\frac{v}{2}}\left(\kappa_{o} r_{o}\right)},
\end{aligned}
$$

where $B$ and $D$ are as previously defined and $E$ is given by

$$
E=\frac{b c\left(w^{2}-1\right)}{A}
$$


Comparing our results for the phase correlation function, $f(\Delta ; \theta, z)$, Eq. (76), with the isotropic spatial correlation function, Eq. (28), we note that

$$
f(\Delta y, \Delta z ; \theta, s)=1-C\left(\sqrt{B(\cos \theta \Delta z)^{2}+2 E \cos \theta \Delta z \Delta y+D(\Delta y)^{2}} ; \nu+1\right)
$$

where $C(\Delta ; \nu+1)$ is $C(r)$ evaluated at $r=\Delta$ with a spectral index $\nu+1$. Using the approximation for $C(r)$ given by Shkarofsky [1968] we note that for $\kappa_{o} \Delta<<1, f(\Delta) \propto \Delta^{2}$, if $\Delta<r_{o}$. On the other hand, if $\Delta>r_{o}$, then

$$
f(\Delta y, \Delta z ; \theta, s) \simeq \frac{1}{\nu 2^{\nu-1}} \frac{\Gamma\left(\frac{2-\nu}{2}\right)}{\Gamma\left(\frac{\nu}{2}\right)}\left(\kappa_{o} \Delta\right)^{\nu}+\frac{1}{2(\nu-2)}\left(\kappa_{o} \Delta\right)^{2} .
$$

The phase correlation function can have two types of behavior for separations between the inner and outer scale depending on whether the spectral index, $\nu$, is less than or greater than 2 . In the former case, the first term on the right-hand side of Eq. (79) dominates so $f \propto \Delta^{\nu}$. But if $\nu>2$ then the second term dominates and $f \propto \Delta^{2}$. The phase correlation function approaches unity as the separation $\Delta$ becomes comparable to the outer scale.

Figure 4 shows $f(\Delta)$ for the case where $\nu=1.65, r_{o}=0.015 \mathrm{~km}$, and $\kappa_{o}=2.5 \mathrm{~km}^{-1}$. As can be seen, $f(\Delta)$ displays two power law behaviors for small separations. As described below, in order to calculate the path integrals for the mutual coherence function for frequency separation it is necessary to invoke a quadratic approximation for $f(\Delta)$; that is

$$
f(\Delta) \sim \frac{1}{2}\left(\frac{\Delta}{L}\right)^{2}
$$

If $\Phi$ is sufficiently large then paths for which $\Delta>\mathrm{L}$ will not contribute to the integrals. In effect, we will restrict ourselves to the Rayleigh fading or fully saturated regime for the mcf of frequency. Thus one may choose an intermediate separation to define the horizontal and vertical correlation lengths. The choice of the intermediate separation depends upon the intensity of the fluctuations. The more intense the smaller the values of $\Delta$ that are important leading to an effective decrease in the correlation length. That is, only separations, $\Delta$, for which $f(\Delta)<1 / \Phi^{2}$ contribute to the path integral. 


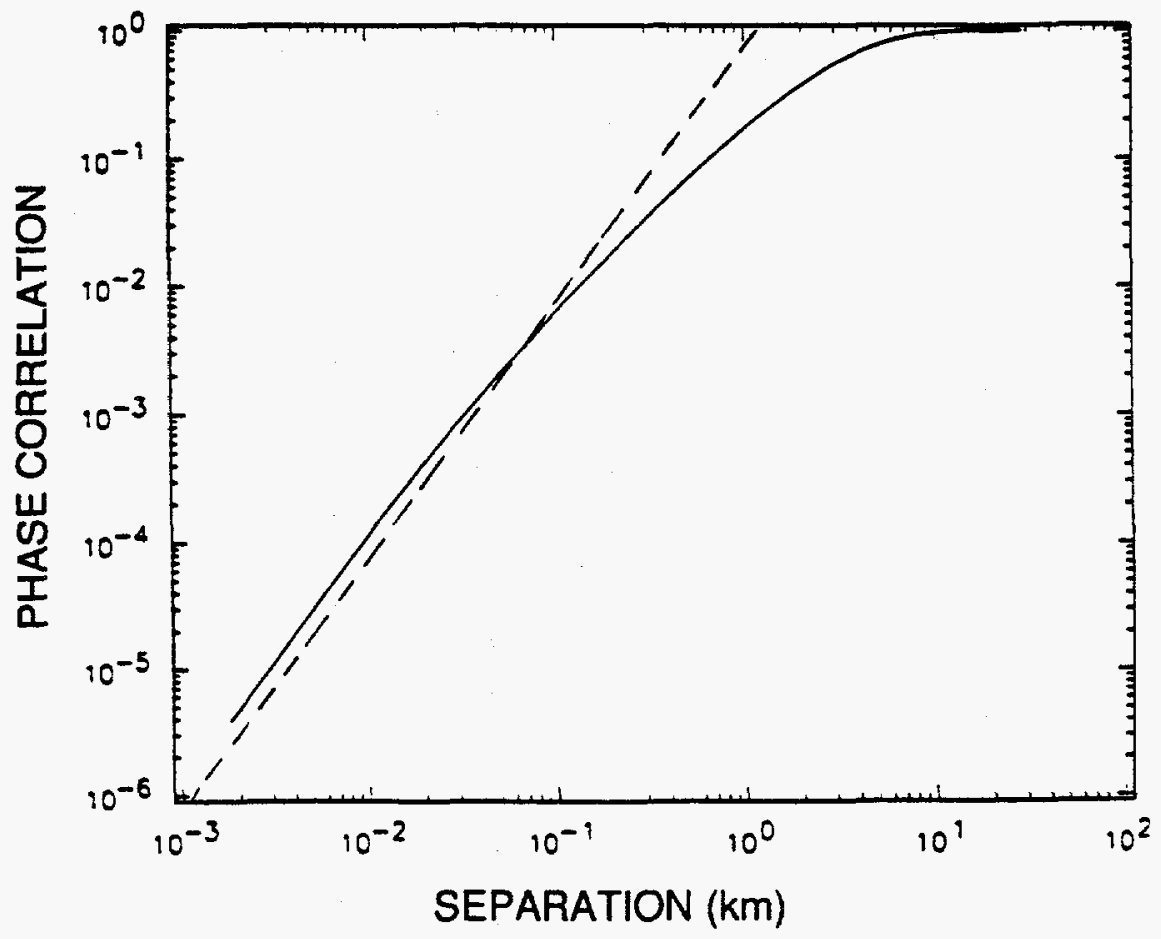

Fig. 4. Behavior of the phase correlation function, $f(\Delta)$, versus separation, $\Delta$, using Eq. (76) with the following parameters: spectral index $=1.65$; outer scale $=2.5 \mathrm{~km}$; inner scale $=15 \mathrm{~m}$; isotropic fluctuations $(w=1)$. Straight line is quadratic approximation to $f(\Delta)$ with a correlation length of $0.85 \mathrm{~km}$.

In general, the correlation function of the irregularities will not be isotropic so that we must seek an approximation for $f(\Delta)$ of the form

$$
f(\Delta y, \Delta z ; \theta, s)=\frac{1}{2}\left(\frac{\Delta y}{L_{\mathrm{H}}}\right)^{2}+\frac{1}{2}\left(\frac{\Delta z}{L_{V}}\right)^{2}-\frac{\Delta y \Delta z}{L_{V \mathrm{H}}^{2}} .
$$

The prescription for evaluating the correlation lengths is to choose $(\Delta y, \Delta z)$ such that $f(\Delta y, \Delta z)=$ $1 / 2 \Phi^{2}$. The transverse correlation length, $L_{\mathrm{H}}$, is evaluated for $\Delta z=0$, the vertical correlation length, $L_{V}$, for $\Delta y=0$. The cross term, $L_{V \mathrm{H}}^{2}$, is then evaluated from the correction term for $\Delta y \neq 0, \Delta z \neq 0$. 
The straight line in Fig. 4 is the quadratic approximation of $f$ with a correlation length $L=0.85 \mathrm{~km}$ valid for $\Phi^{2}=100$. Figure 5 shows the behavior of the correlation length versus the intensity of the fluctuations, $\Phi^{2}$. With increasing $\Phi^{2}$ the correlation approaches asymptotically the length valid for separations within the inner scale.

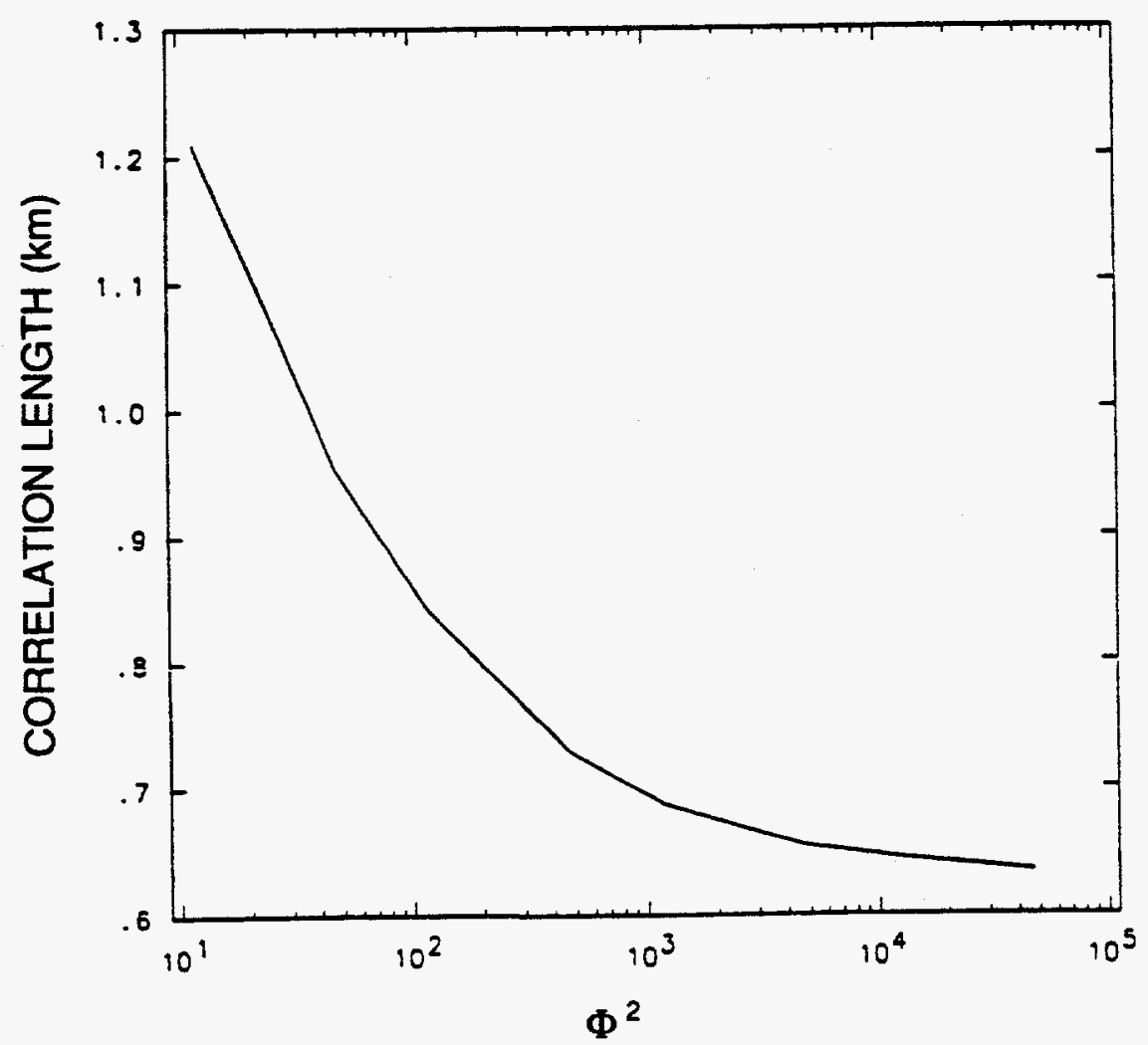

Fig. 5. Dependence of the correlation length used in the quadratic approximation to the phase correlation function Eq. (80), upon the strength of the fluctuations given by the variance of the phase, $\Phi^{2}$. Parameters are the same as those used in Figure 4.

\subsection{Mutual coherence function for time separations}

The mutual coherence of a single receiver versus time delay, $\Delta t$, is from Eq. (56)

$$
\left\langle\psi^{*}(\Delta t) \psi(0)\right\rangle=\exp \left[-\frac{1}{2} D(\Delta t)\right],
$$


where the phase structure function is given by

$$
D(\Delta t)=2 k_{o}^{2} \int d s\left\langle\mu^{2}(s)\right\rangle L_{p}(\theta, s) f(\Delta t ; \theta, s)
$$

Let us assume that the spatial fluctuations are transported transverse to the ray path at an average velocity $v_{t}$. Transport parallel to the ray path may be ignored because the rays see the same fluctuations. Vertical transport is usually smaller than horizontal transport. Thus the phase correlation function, $f(\Delta t ; \theta, s)$, is

$$
f(\Delta t ; \theta, s)=f\left(\Delta y=v_{t} \Delta t, \Delta z=0 ; \theta, s\right)
$$

so that the mcf versus time delay, Eq. (82) may be numerically integrated in the same manner as for receiver separation. It would be the same as the mcf for receiver separation $\Delta y=v_{t} \Delta t$ except that, since most of the contribution comes from the middle of the path while $\Delta y$ is at the end of the path, one should use an effective transport velocity twice as great as the actual velocity. Also, the mcf for horizontal separation employs a difference in azimuth and thus a variable separation over the path but the mcf for time delay should be calculated at a constant separation. For a long path with a limited extent in the ionosphere, the latter correction will be small.

If instead of using the uniform transport model for the time dependence of the correlation function, we use the decorrelation time scale embodied in Eq. (25) then Eq. (64) becomes

$$
f(\Delta t ; \theta, s)=1-\frac{1}{L_{p}(\theta, s)} \int d s^{\prime} C\left(s, s^{\prime}\right) e^{-\frac{1}{2}\left(\frac{\Delta t}{T}\right)^{2}}
$$

where the integral is along the same ray path. Using the definition of $L_{p}$, Eq. (47), we obtain

$$
f(\Delta t ; \theta, s)=1-e^{-\frac{1}{2}\left(\frac{\Delta t}{T}\right)^{2}}
$$

Substituting this expression into Eq. (83) and using Eq. (48) we obtain

$$
\left\langle\psi^{*}(\Delta t) \psi(0)\right\rangle=\exp \left\{-k_{0}^{2} \Phi^{2}\left[1-\exp \left(-\frac{1}{2}\left(\frac{\Delta t}{T}\right)^{2}\right)\right]\right\} .
$$


The mcf versus time delay, $\left\langle\psi^{*}(\Delta t) \psi(0)\right\}$, may be looked upon as the autocorrelation of a $\mathrm{cw}$ transmission. Its Fourier transform is the average Doppler spectrum of the transmission.

\section{MUTUAL COHERENCE FUNCTION VERSUS FREQUENCY}

Given a single receiver we wish to calculate the mutual coherence function of two transmissions at different frequencies [Dashen, Flatté, and Reynolds, 1985]. That is, we consider only frequency separations and neither space or time separations. The mcf versus frequency will define the coherent bandwidth of the transmission. The effect of random electron density fluctuations in the ionosphere is to decorrelate the two rays which follow slightly different nominal paths because of the frequency dependence of the background refractive index.

The mcf at frequencies $\omega_{1}$ and $\omega_{2}$ is

$$
\left\langle\psi^{*}(1) \psi(2)\right\rangle=|\mathcal{N}|^{2} \int D \mathbf{z}_{1} D \mathbf{z}_{2} \exp \left[i k_{1} S_{o}(1)-i k_{2} S_{o}(2)-\frac{1}{2} V_{12}\right]
$$

where $k_{1}=\omega_{1} / c_{o}$ and $k_{2}=\omega_{2} / c_{o}$. The effect of the fluctuations is included in the $V_{12}$ term:

$$
V_{12}=\left\langle\left(k_{1} \int_{0}^{R} \mu\left(\mathbf{z}_{1}, k_{1}\right) d x-k_{2} \int_{0}^{R} \mu\left(\mathbf{z}_{2}, k_{2}\right) d x\right)^{2}\right\rangle
$$

where we have used

$$
\langle\exp (i \alpha)\rangle=\exp \left(-\frac{1}{2}\left\langle\alpha^{2}\right\rangle\right)
$$

The unperturbed phase difference is given by

$$
\begin{aligned}
k_{1} S_{o}(1)-k_{2} S_{o}(2)=\int_{o}^{R}\left[\frac{1}{2} k_{1}\left(\frac{\partial y_{1}}{\partial x}\right)^{2}\right. & -\frac{1}{2} k_{2}\left(\frac{\partial y_{2}}{\partial x}\right)^{2}+\frac{1}{2} k_{1}\left(\frac{\partial z_{1}}{\partial x}\right)^{2}-\frac{1}{2} k_{2}\left(\frac{\partial z_{2}}{\partial x}\right)^{2} \\
& \left.-k_{1} U_{o}\left(z_{1}, k_{1}\right)+k_{2} U_{o}\left(z_{2}, k_{2}\right)\right] d x
\end{aligned}
$$

We define new variables

$$
\alpha(x)=y_{1}(x)-y_{2}(x)
$$

and

$$
\beta(x)=\frac{1}{2}\left[y_{1}(x)+y_{2}(x)\right]
$$


so that

$$
\frac{1}{2} k_{1}\left(\frac{\partial y_{1}}{\partial x}\right)^{2}-\frac{1}{2} k_{2}\left(\frac{\partial y_{2}}{\partial x}\right)^{2}=\bar{k} \frac{\partial \alpha}{\partial x} \frac{\partial \beta}{\partial x}+\frac{1}{2} \Delta k\left[\frac{1}{4}\left(\frac{\partial \alpha}{\partial x}\right)^{2}+\left(\frac{\partial \beta}{\partial x}\right)^{2}\right],
$$

where $\Delta k=k_{1}-k_{2}$ and $\bar{k}=\frac{1}{2}\left(k_{1}+k_{2}\right)$. Define $z_{r}(x)$ as the nominal ray path at frequency $\bar{\omega}=c_{o} \bar{k}$ and

$$
v_{1}(x)=z_{1}(x)-z_{r}(x)
$$

and

$$
v_{2}(x)=z_{2}(x)-z_{r}(x)
$$

The nominal ray path in the parabolic approximation is a solution of

$$
\frac{\partial^{2} z_{r}}{\partial x^{2}}+U_{o}^{\prime}\left(z_{r}, \bar{k}\right)=0
$$

where the prime indicates the first derivative [Flatté et al., 1979].

Using the new variables, $v_{i}(i=1,2)$, we may approximate $U_{o}\left(z_{i}, k_{i}\right)$ as

$$
U_{o}\left(z_{i}, k_{i}\right)=U\left(z_{r}\right)+U^{\prime}\left(z_{r}\right) v_{i}+\frac{1}{2} U^{\prime \prime}\left(z_{r}\right) v_{i}^{2}-(-1)^{i}\left(U_{k}+U_{k}^{\prime} v_{i}\right) \frac{\Delta k}{2}+\frac{1}{2} U_{k k}\left(\frac{\Delta k}{2}\right)^{2} \text {, }
$$

where the subscript $k$ indicates a derivative with respect to $k$ and we drop the subscript 0 . Then

$$
\begin{aligned}
k_{1} U_{o}\left(z_{1}, k_{1}\right)-k_{2} U_{o}\left(z_{2}, k_{2}\right)=\Delta k U\left(z_{r}\right) & +U^{\prime}\left(k_{1} v_{1}-k_{2} v_{2}\right)+\frac{1}{2} U^{\prime \prime}\left(k_{1} v_{1}^{2}-k_{2} v_{2}^{2}\right) \\
& +U_{k} \bar{k} \Delta k+U_{k}^{\prime} \frac{\Delta k}{2}\left(k_{1} v_{1}+k_{2} v_{2}\right)+U_{k k}\left(\frac{\Delta k}{2}\right)^{3}
\end{aligned}
$$

Defining another set of variables

$$
u(x)=v_{1}(x)-v_{2}(x)
$$

and

$$
w(x)=\frac{1}{2}\left[v_{1}(x)+v_{2}(x)\right]
$$

one finds that

$$
\begin{aligned}
k_{1} U_{o}\left(z_{1}, k_{1}\right) & -k_{2} U_{o}\left(z_{2}, k_{2}\right)=\bar{k}\left(U^{\prime} u+U^{\prime \prime} u w+\Delta k U_{k}+\Delta k U_{k}^{\prime} w\right) \\
& +\frac{1}{2} \Delta k\left[2 U+2 U^{\prime} w+U^{\prime \prime} w^{2}+U^{\prime \prime} \frac{u^{2}}{4}+\frac{\Delta k}{2} U_{k}^{\prime} u+U_{k k}\left(\frac{\Delta k}{2}\right)^{2}\right]
\end{aligned}
$$


Since

$$
z_{1}=z_{r}+w+\frac{u}{2}
$$

and

$$
\begin{gathered}
z_{2}=z_{r}+w-\frac{u}{2} \\
\frac{1}{2} k_{1}\left(\frac{\partial z_{1}}{\partial x}\right)^{2}-\frac{1}{2} k_{2}\left(\frac{\partial z_{2}}{\partial x}\right)^{2}=\frac{1}{2} \Delta k\left[\left(\frac{\partial z_{r}}{\partial x}\right)^{2}+2 \frac{\partial z_{r}}{\partial x} \frac{\partial w}{\partial x}+\left(\frac{\partial w}{\partial x}\right)^{2}+\frac{1}{4}\left(\frac{\partial u}{\partial x}\right)^{2}\right] \\
+\bar{k}\left[\frac{\partial z_{r}}{\partial x} \frac{\partial u}{\partial x}+\frac{\partial w}{\partial x} \frac{\partial u}{\partial x}\right] .
\end{gathered}
$$

Substituting Eqs. (94), (102), and (105) into Eq. (91) we have

$$
\begin{aligned}
k_{1} S_{o}(1) & -k_{2} S_{o}(2)=\bar{k} \int_{o}^{R} d x\left[\frac{\partial \alpha}{\partial x} \frac{\partial \beta}{\partial x}-U^{\prime} u-U^{\prime \prime} u w+\frac{\partial z_{r}}{\partial x} \frac{\partial u}{\partial x}+\frac{\partial w}{\partial x} \frac{\partial u}{\partial x}-U_{k} \Delta k-U_{k}^{\prime} \Delta k w\right] \\
& +\frac{1}{2} \Delta k \int_{o}^{R} d x\left[\left(\frac{\partial \beta}{\partial x}\right)^{2}+\frac{1}{4}\left(\frac{\partial \alpha}{\partial x}\right)^{2}+\left(\frac{\partial z_{r}}{\partial x}\right)^{2}+2 \frac{\partial z_{r}}{\partial x} \frac{\partial w}{\partial x}+\left(\frac{\partial w}{\partial x}\right)^{2}\right. \\
& \left.+\frac{1}{4}\left(\frac{\partial u}{\partial x}\right)^{2}-2 U-2 U^{\prime} w-U^{\prime \prime} w^{2}-\frac{1}{4} U^{\prime \prime} u^{2}-U_{k}^{\prime} \frac{\Delta k}{2} u-U_{k k}\left(\frac{\Delta k}{2}\right)^{2}\right]
\end{aligned}
$$

We notice that four of the integrands in Eq. (106) do not depend on the path variables but contribute a deterministic phase, $\phi_{d}$, to the mutual coherence function. That is

$$
\phi_{d}=\frac{\Delta k}{2} \int_{0}^{R}\left[\left(\frac{\partial z_{r}}{\partial x}\right)^{2}-2 U-2 \bar{k} U_{k}-\left(\frac{\Delta k}{2}\right)^{2} U_{k k}\right] d x .
$$

The term proportional to $\Delta k$ in this deterministic phase shift may be interpreted in terms of group delay; it contains a factor proportional to the path difference between the refracted ray and the direct ray and another factor arising from dispersion which represents the difference of phase and group velocities. The higher order term represents a variable delay versus frequency and produces pulse spreading due to dispersion. Since these effects are deterministic we will not consider them further.

We assume that $V_{12}$, defined by Eq. (89), is a function of the path separation, $\mathbf{z}_{1}-\mathbf{z}_{2}$, and thus does not depend on the average location of the paths incorporated in the variables $\beta$ and $w$. The path integral, $I_{\beta}$, over the variable $\beta$ is

$$
I_{\beta}=\int D \beta \exp \left[\frac{i}{2} \Delta k \int_{0}^{R}\left(\left(\frac{\partial \beta}{\partial x}\right)^{2}+\frac{2 \bar{k}}{\Delta k} \frac{\partial \alpha}{\partial x} \frac{\partial \beta}{\partial x}\right) d x\right] .
$$


Completing the square, one obtains

$$
I_{\beta}=\exp \left(-\frac{i}{2} \frac{\bar{k}^{2}}{\Delta k} \int_{o}^{R}\left(\frac{\partial \alpha}{\partial x}\right)^{2} d x\right) \int D \beta \exp \left\{\frac{i}{2} \Delta k \int_{o}^{R}\left[\frac{\partial}{\partial x}\left(\beta+\frac{\bar{k}}{\Delta k} \alpha\right)\right]^{2} d x\right\}
$$

By changing the integration variable $\beta=\beta+\frac{\bar{k}}{\Delta k} \alpha$ we observe that the path integral in Eq. (109) is a simple factor which can be incorporated into the normalization.

The path integral, $I_{w}$, over $w$ is

$$
\begin{aligned}
I_{w}=\int D w \exp \left\{\frac{i}{2} \Delta k\right. & \int_{o}^{R}\left[2 \frac{\partial z_{\tau}}{\partial x} \frac{\partial w}{\partial x}+\left(\frac{\partial w}{\partial x}\right)^{2}-2 U^{\prime} w-U^{\prime \prime} w^{2}\right. \\
& \left.\left.+\frac{2 \bar{k}}{\Delta k}\left(-U^{\prime \prime} u w+\frac{\partial w}{\partial x} \frac{\partial u}{\partial x}-U_{k}^{\prime} \Delta k w\right)\right] d x\right\}
\end{aligned}
$$

Using the fact that $w(0)=w(R)=0$ and $u(0)=u(R)=0$ one can show that

$$
\begin{array}{r}
\int_{0}^{R} \frac{\partial w}{\partial x} \frac{\partial u}{\partial x} d x=-\int_{0}^{R} w \frac{\partial^{2} u}{\partial x^{2}} d x \\
\int_{0}^{R} \frac{\partial w}{\partial x} \frac{\partial u}{\partial x} d x=-\int_{0}^{R} u \frac{\partial^{2} w}{\partial x^{2}} d x \\
\int_{0}^{R}\left(\frac{\partial w}{\partial x}\right)^{2} d x=-\int_{0}^{R} w \frac{\partial^{2} w}{\partial x^{2}} d x
\end{array}
$$

and

$$
\int_{0}^{R} 2 \frac{\partial z_{r}}{\partial x} \frac{\partial w}{\partial x} d x=-\int_{0}^{R} 2 w \frac{\partial^{2} z_{r}}{\partial x^{2}} d x
$$

Using Eq. (97) one notes that

$$
-\int_{0}^{R} 2 w \frac{\partial^{2} z_{r}}{\partial x^{2}} d x-\int_{0}^{R} 2 U^{\prime} w d x=0
$$

Defining the operator $L=-\frac{\partial^{2}}{\partial x^{2}}-U^{\prime \prime}, I_{w}$ becomes

$$
I_{w}=\int D w \exp \left\{\frac{i}{2} \Delta k \int_{o}^{R}\left[\left(w L w+\frac{\bar{k}}{\Delta k} w L u+\frac{\bar{k}}{\Delta k} u L w\right)-2 \bar{k} U_{k}^{\prime} w\right] d x\right\} .
$$

Completing the square, one finds that

$$
\begin{aligned}
I_{w} & =\exp \left(-i \frac{\Delta k}{2} \int_{0}^{R}\left[\frac{\bar{k}^{2}}{(\Delta k)^{2}} u L u-\frac{2 \bar{k}^{2} U_{k}^{\prime} u}{\Delta k}\right] d x\right) \\
& \times \int D w \exp \left\{\frac{i}{2} \Delta k \int_{o}^{R}\left[\left(w+\frac{\bar{k}}{\Delta k} u\right) L\left(w+\frac{\bar{k}}{\Delta k} u\right)-2 \bar{k} U_{k}^{\prime}\left(w+\frac{\bar{k}}{\Delta k} u\right)\right] d x\right\} .
\end{aligned}
$$


Changing the integration variable $w=w+\frac{\bar{k}}{\Delta k} u$ the path integral in Eq. (117) is just a factor that can be subsumed into the normalization.

The remaining terms in Eq. (106) can be written as

$$
\bar{k} \int_{0}^{R}\left[-U^{\prime} u+\frac{\partial z_{r}}{\partial x} \frac{\partial u}{\partial x}\right] d x+\frac{\Delta k}{2} \int_{0}^{R}\left[\frac{1}{4}\left(\frac{\partial \alpha}{\partial x}\right)^{2}+\frac{1}{4}\left(\frac{\partial u}{\partial x}\right)^{2}-\frac{1}{4} U^{\prime \prime} u^{2}-U_{k}^{\prime} \frac{\Delta k}{2} u\right] d x
$$

Using the same argument as in Eq. (112) the first integral is zero. The second integral can be written as

$$
\frac{\Delta k}{2} \int_{o}^{R}\left[\frac{1}{4}\left(\frac{\partial \alpha}{\partial x}\right)^{2}+\frac{1}{4} u L u-U_{k}^{\prime} \frac{\Delta k}{2} u\right] d x
$$

Combining Eqs. (109), (117), and (119) we obtain for the mcf

$$
\begin{gathered}
\left\langle\psi^{*}\left(k_{2}\right) \psi\left(k_{1}\right)\right\rangle=\mathcal{N} \int D \alpha D u \exp \left\{-\frac{i}{2} \frac{\bar{k}^{2}}{\Delta k} \int_{o}^{R}\left[\left(\frac{\partial \alpha}{\partial x}\right)^{2}+u L u\right] d x+\frac{i}{2} \Delta k \int_{o}^{R}\left[\frac{1}{4}\left(\frac{\partial \alpha}{\partial x}\right)^{2}+\right.\right. \\
\left.\left.\frac{1}{4} u L u+U_{k}^{\prime} u\left(\frac{2 \bar{k}^{2}}{\Delta k}-\frac{\Delta k}{2}\right)\right] d x-\frac{1}{2} V_{12}\right\}
\end{gathered}
$$

or

$$
\begin{aligned}
\left\langle\psi^{*}\left(k_{2}\right) \psi\left(k_{1}\right)\right\rangle & =\mathcal{N} \int D \alpha D u \exp \left\{-\frac{i}{2} \frac{k_{1} k_{2}}{\Delta k} \int_{o}^{R}\left[\left(\frac{\partial \alpha}{\partial x}\right)^{2}+u L u\right] d x\right. \\
& \left.+\frac{i}{2} \Delta k \int_{o}^{R} U_{k}^{\prime} u\left(\frac{2 \bar{k}^{2}}{\Delta k}-\frac{\Delta k}{2}\right) d x-\frac{1}{2} V_{12}\right\}
\end{aligned}
$$

Turning to the $V_{12}$ term, we can rewrite Eq. (89) as

$$
\begin{aligned}
V_{12} & =\left\langle\left\{\left(\bar{k}+\frac{\Delta k}{2}\right) \int_{o}^{R}\left[\mu\left(z_{1}, \bar{k}\right)+\frac{\partial \mu\left(z_{1}\right)}{\partial k} \frac{\Delta k}{2}\right] d x\right.\right. \\
& \left.\left.-\left(\bar{k}-\frac{\Delta k}{2}\right) \int_{0}^{R}\left[\mu\left(z_{2}, \bar{k}\right)-\frac{\partial \mu\left(z_{2}\right)}{\partial k} \frac{\Delta k}{2}\right] d x\right\}^{2}\right\rangle
\end{aligned}
$$

If the high frequency approximation, Eq. (23), is used in Eq. (12) for $\mu$ one obtains

$$
\mu=\frac{1}{2} \frac{\omega_{o}^{2}}{\omega^{2}} \frac{\Delta N}{N}
$$

from which one can show that

$$
\frac{\partial \mu}{\partial k}=-\frac{2}{\omega} \frac{\partial \omega}{\partial k} \mu .
$$


We recall that $k$ is the free space wave number so that

$$
\frac{\partial \mu}{\partial k}=-\frac{2 \mu}{k}
$$

Using this approximation for the derivatives in Eq. (122), one obtains for $V_{12}$ after some algebra

$$
\begin{aligned}
V_{12} & =\left\langle\left\{\bar{k}\left[1-\frac{1}{2}\left(\frac{\Delta k}{\bar{k}}\right)^{2}\right]\left[\int_{0}^{R} \mu\left(z_{1}, \bar{k}\right) d x-\int_{0}^{R} \mu\left(z_{2}, \bar{k}\right) d x\right]\right.\right. \\
& \left.\left.-\frac{\Delta k}{2}\left[\int_{0}^{R} \mu\left(z_{1}, \bar{k}\right) d x+\int_{0}^{R} \mu\left(z_{2}, \bar{k}\right) d x\right]\right\}^{2}\right\rangle .
\end{aligned}
$$

We assume that the rms phase deviation, $\Phi^{2}$, where

$$
\Phi^{2}=\bar{k}^{2}\left\langle\left(\int_{0}^{R} \mu(z, \bar{k}) d x\right)^{2}\right\rangle
$$

is independent of path; then Eq. (126) becomes

$$
\begin{aligned}
V_{12} & =\bar{k}^{2}\left[1-\frac{1}{2}\left(\frac{\Delta k}{\bar{k}}\right)^{2}\right]^{2}\left\langle\left(\int_{0}^{R} \mu\left(z_{1}, \bar{k}\right) d x-\int_{0}^{R} \mu\left(z_{2}, \bar{k}\right) d x\right)^{2}\right\rangle \\
& +\left(\frac{\Delta k}{2}\right)^{2}\left\langle\left(\int_{0}^{R} \mu\left(z_{1}, \bar{k}\right) d x+\int_{0}^{R} \mu\left(z_{2}, \bar{k}\right) d x\right)^{2}\right\rangle .
\end{aligned}
$$

The first term on the right hand side of Eq. (128) is simply proportional to the phase structure function, $D(1,2)$, defined in Eq. (57) and given in terms of the phase correlation function, $f$, in Eq. (62). The second term on the right hand side can also be related to the phase structure function, so that one finds

$$
V_{12}=2 \bar{k}^{2}\left[1-\frac{1}{2}\left(\frac{\Delta k}{\bar{k}}\right)\right]^{2} \int_{o}^{R}\left\langle\mu^{2}\right\rangle L_{p} f(\alpha, u, 0) d x+\left(\frac{\Delta k}{2}\right)^{2}\left[\frac{4 \Phi^{2}}{\bar{k}^{2}}-2 \int_{o}^{R}\left\langle\mu^{2}\right\rangle L_{p} f(\alpha, u, 0) d x\right]
$$

or approximately

$$
V_{12}=\left(\frac{\Delta k}{\bar{k}}\right)^{2} \Phi^{2}+2 \bar{k}^{2}\left[1-\frac{5}{4}\left(\frac{\Delta k}{\bar{k}}\right)^{2}\right] \int_{0}^{R}\left\langle\mu^{2}\right\rangle L_{p} f(\alpha, u, o) d x
$$

With this formulation of $V_{12}$, the mutual coherence function may be written as

$$
\left\langle\psi^{*}\left(k_{2}\right) \psi\left(k_{1}\right)\right\rangle=\exp \left[-\frac{1}{2}\left(\frac{\Delta k}{\bar{k}}\right)^{2} \Phi^{2}\right] Q(\Delta k)
$$


where

$$
\begin{aligned}
Q(\Delta k) & =\mathcal{N} \int D \alpha D u \exp \left\{-\frac{i}{2} \frac{k_{1} k_{2}}{\Delta k} \int_{o}^{R}\left[\left(\frac{\partial \alpha}{\partial x}\right)^{2}+u L u\right] d x+\frac{i}{2} \Delta k\left(\frac{2 \bar{k}^{2}}{\Delta k}-\frac{\Delta k}{2}\right) \int_{o}^{R} U_{k}^{\prime} u d x\right. \\
& \left.-\bar{k}^{2}\left[1-\frac{5}{4}\left(\frac{\Delta k}{\bar{k}}\right)^{2}\right] \int_{o}^{R}\left\langle\mu^{2}\right\rangle L_{p} f(\alpha, u, o) d x\right\}
\end{aligned}
$$

Assuming $k_{1} k_{2}=\bar{k}^{2}, \bar{k} \gg \Delta k$, and expanding $L$ we obtain

$$
\begin{aligned}
Q(\Delta k) & =\mathcal{N} \int D \alpha D u \exp \left\{-\frac{i}{2} \frac{\bar{k}^{2}}{\Delta k} \int_{0}^{R}\left[\left(\frac{\partial \alpha}{\partial x}\right)^{2}+\left(\frac{\partial u}{\partial x}\right)^{2}-U^{\prime \prime} u^{2}-2 U_{k}^{\prime} \Delta k u\right.\right. \\
& \left.\left.-2 i \Delta k\left\langle\mu^{2}\right\rangle L_{p} f(\alpha, u, o)\right] d x\right\}
\end{aligned}
$$

In this two-dimensional path integral we may identify the analog of the Lagrangian as

$$
G=-\frac{1}{2} \frac{\bar{k}^{2}}{\Delta k}\left[\left(\frac{\partial \alpha}{\partial x}\right)^{2}+\left(\frac{\partial u}{\partial x}\right)^{2}-U^{\prime \prime} u^{2}-2 U_{k}^{\prime} \Delta k u-F\right]
$$

where $F=2 i \Delta k\left\langle\mu^{2}\right\rangle L_{p} f(\alpha, u, o)$.

The semiclassical approximation to the solution of the two-dimensional path integral may be obtained in the regime for which the quadratic approximation for $f$ is valid, that is, $\Phi \gg 1$. Schulman [1981] shows using the quadratic approximation that

$$
Q(\Delta k) \propto\left[\operatorname{det} J_{i k}(R)\right]^{-1 / 2} \exp \left(i S_{c}\right)
$$

where $J_{i k}(x)$ satisfies Jacobi equations

$$
\frac{d}{d x}\left(\frac{\partial^{2} G}{\partial \dot{q}_{i} \partial \dot{q}_{l}} \dot{J}_{l k}\right)+\left(\frac{\partial^{2} G}{\partial \dot{q}_{i} \partial q_{l}}-\frac{\partial^{2} G}{\partial q_{i} \partial \dot{q}_{l}}\right) \dot{J}_{l k}+\left[\frac{d}{d x}\left(\frac{\partial^{2} G}{\partial \dot{q}_{i} \partial q_{l}}\right)-\frac{\partial^{2} G}{\partial q_{i} \partial q_{l}}\right] J_{l k}=0
$$

and $S_{c}$ is the action along the classical path, that is, the path for which the action is an extremum. In Eq. (136) the dot indicates a derivative with respect to $x, q=(\alpha, u)$ and there is an implied sum over doubled indices. The initial conditions are

$$
\begin{gathered}
J_{i k}(0)=0, \\
\frac{d J_{i k}(0)}{d x}=\delta_{i k} .
\end{gathered}
$$


When Eq. (136) is evaluated with $G$ given by Eq. (134) we obtain

$$
\left[\begin{array}{cc}
2 \frac{d^{2}}{d x^{2}}+\frac{\partial^{2} F}{\partial \alpha^{2}} & \frac{\partial^{2} F}{\partial \alpha \partial u} \\
\frac{\partial^{2} F}{\partial \alpha \partial u} & 2 \frac{d^{2}}{d x^{2}}+2 U_{o}^{\prime \prime}+\frac{\partial^{2} F}{\partial u^{2}}
\end{array}\right] \quad\left[\begin{array}{ll}
J_{11} & J_{12} \\
J_{21} & J_{22}
\end{array}\right]=0
$$

The classical path is a solution of Lagrange's equations which for Eq. (134) may be written as

$$
2 \frac{\partial^{2} \alpha}{\partial x^{2}}+\frac{\partial F}{\partial \alpha}=0
$$

and

$$
2 \frac{\partial^{2} u}{\partial x^{2}}+2 U^{\prime \prime} u+2 U_{k}^{\prime} \Delta k+\frac{\partial F}{\partial u}=0
$$

with the boundary conditions $u(0)=u(R)=\alpha(0)=\alpha(R)=0$. The solution to Eq. (139) and Eq. (140) when $F$ is complex may be obtained by analytic continuation into a complex path [Koeling and Malfiet, 1975]. If the dispersion term in Eq. (140) were not present, the equations would have the trivial solution $\alpha(x)=u(x)=0$. The inclusion of dispersion causes the path, chiefly the vertical displacement $u$, to be displaced from the nominal ray. That is, Eq. (10) may be viewed as the linearized perturbation expansion of the equation for the ray path, Eq. (97). Thus the inclusion of dispersion causes the action, $S_{c}$, to be complex so that the magnitude of $Q(\Delta k)$ in Eq. (135) is reduced. Essentially, the loss of coherence due to dispersion is proportional to the ratio of the displacement of the ray to the correlation lengths of the fluctuations. Moreover, the phase of $Q$ versus $\Delta k$ will depend on the strength of the fluctuations since that determines how far off the real axis $u(x)$ and $\alpha(x)$ are. We choose a normalization such that $Q(0)=1$ so that

$$
Q(\Delta k)=\left[\frac{\operatorname{det} J_{i k}(R, 0)}{\operatorname{det} J_{i k}(R, \Delta k)}\right]^{1 / 2} \exp \left[i S_{c}(\Delta k)\right]
$$

In the limit as $\Delta k \rightarrow 0, Q(\Delta \omega)$ - where $\Delta \omega=c \Delta k$ - may be written as

$$
Q(\Delta \omega)=\exp \left[-\frac{1}{2}\left(\Delta \omega \tau_{o}\right)^{2}+i \Delta \omega \tau_{1}\right]
$$

which using the properties of Fourier transforms, indicates that the fluctuations produce a mean time delay, $\tau_{1}$, and a Gaussian spread, $\tau_{0}$. In general, the ensemble average pulse will be not be 
symmetric but skewed as indicated by a nonlinear behavior of the phase of $Q$ as a function of frequency separation, $\Delta \omega$.

\section{SAMPLE RESULTS}

As an example of the effects predicted by the present formalism we will present numerical results for a $1950 \mathrm{~km}$ path in the polar region similar to that reported by Basler et al., [1988]. The ionosphere is modelled as a single nighttime $\mathrm{F}$ layer with a critical frequency of $5.1 \mathrm{MHz}$ at a height of $310 \mathrm{~km}$. The electron density profile is assumed to be a Chapman layer with a scale height of $37 \mathrm{~km}$. Figure 6 shows the ray path of an $11.1 \mathrm{MHz}$ transmission in the rectangular coordinate system used for the calculation; the curved arcs indicate the surface of the earth and the altitude one scale height below the layer maximum. The low-ray, which we consider here, has an elevation angle with respect to the earth's surface of $12.55^{\circ}$. The ray path is oriented magnetic east-west; we assume a dip angle of $80^{\circ}$ for the field.

For this example we assume that the variance of the relative electron density fluctuations is independent of altitude and equal to 0.01 . The density fluctuations follow the Shkarofsky distribution given by Eq. (28) with an outer scale $\left(1 / \kappa_{o}\right)$ of $10 \mathrm{~km}$, a spectral index of 1.65 , an inner scale of $0.01 \mathrm{~km}$, and an elongation of $w=0.1$. For these parameters, the parallel correlation length, $L_{p}$,

is $10.4 \mathrm{~km}$ while the variance of the phase fluctuations, $\Phi^{2}$, is $4160 \mathrm{rad}^{2}$. The correlation lengths used in the approximation to the phase correlation function given by Eq. (81) are $10.2 \mathrm{~km}$ in the vertical direction, $2.1 \mathrm{~km}$ in the horizontal direction, and $5.0 \mathrm{~km}$ for the cross direction $\left(L_{V H}\right)$. The relatively small correlation lengths illustrate the decrease in effective scale lengths with increasing fluctuation strength as shown in Figure 5. The relatively large correlation length in the vertical direction is a consequence of the elongation of the structure along the steeply sloping magnetic field.

The calculated mutual coherences for receiver separations in the transverse and vertical directions are shown in Figure 7. In the transverse direction, the coherence length is approximately 


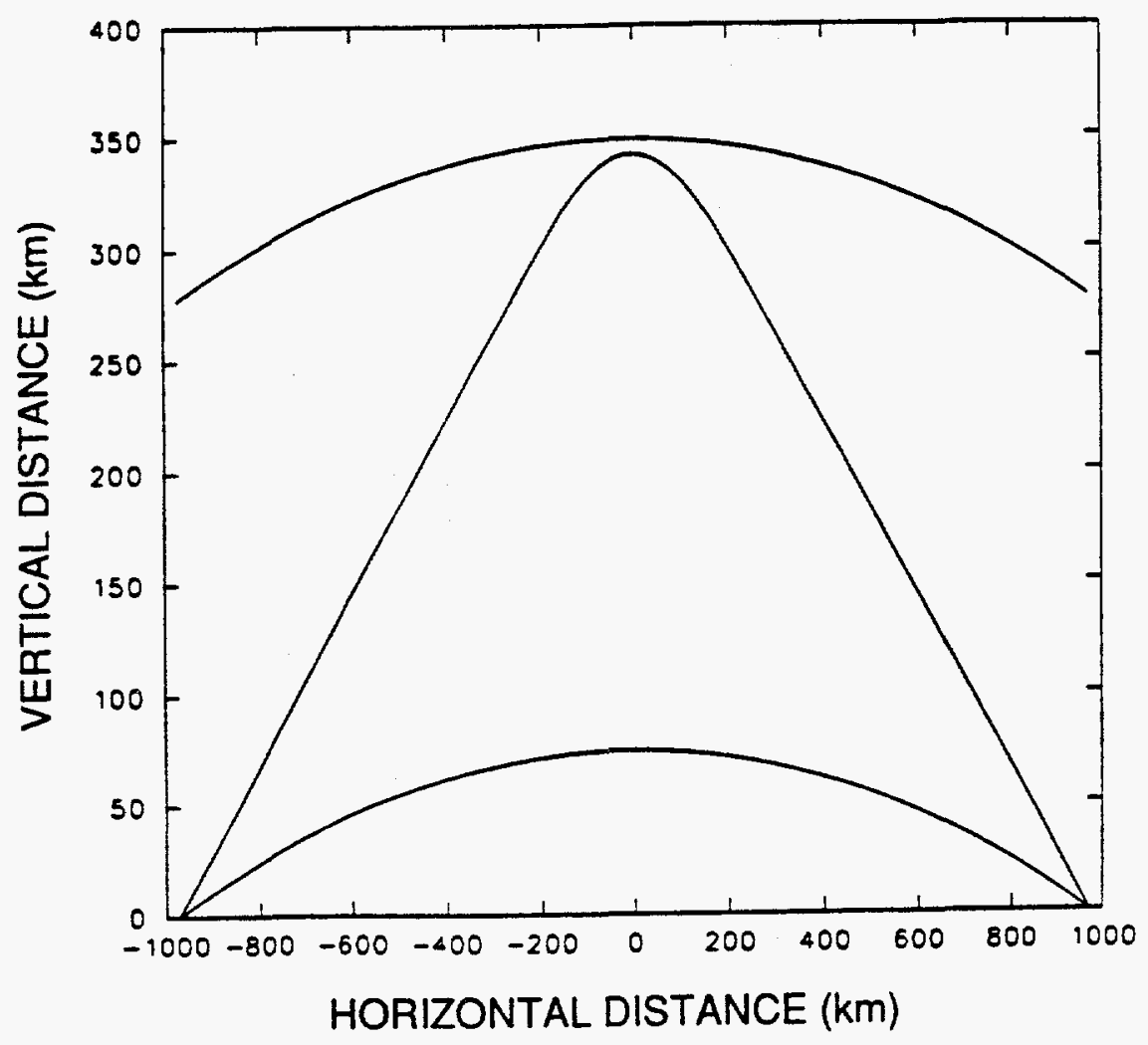

Fig. 6. Raypath (middle curve) in rectangular coordinates used for numerical simulation. We assume a single layer, Chapman-profile, electron density distribution with a maximum at $312 \mathrm{~km}$ altitude. The lower curve represents the surface of the earth; upper curve is at an altitude of one scale height below that of the maximum.

$80 \mathrm{~m}$ while in the vertical direction it is approximately $900 \mathrm{~m}$. The difference in coherence lengths for the two directions reflects the fact that the structures are elongated in the vertical direction. The angular spread of the received signal in the transverse direction, given the calculated coherence length, corresponds to a half angle of approximately $10^{\circ}$. If the antenna directivity is uniform over that angular range, then our assumption of isotropic antenna patterns would be valid.

The results of the simulation for the frequency coherence are shown in Figure 8 where we estimate a half bandwidth of $20 \mathrm{kHz}$. The ensemble average pulse response is illustrated in Figure 9 where we assume a transmitted pulse of Gaussian profile with a full width at half maximum of $10 \mu \mathrm{s}$. The average delay, $\tau_{1}$, is $5.9 \mu$ s while the average spread, $\tau_{0}$, is $11.5 \mu \mathrm{s}$. These results depend greatly 


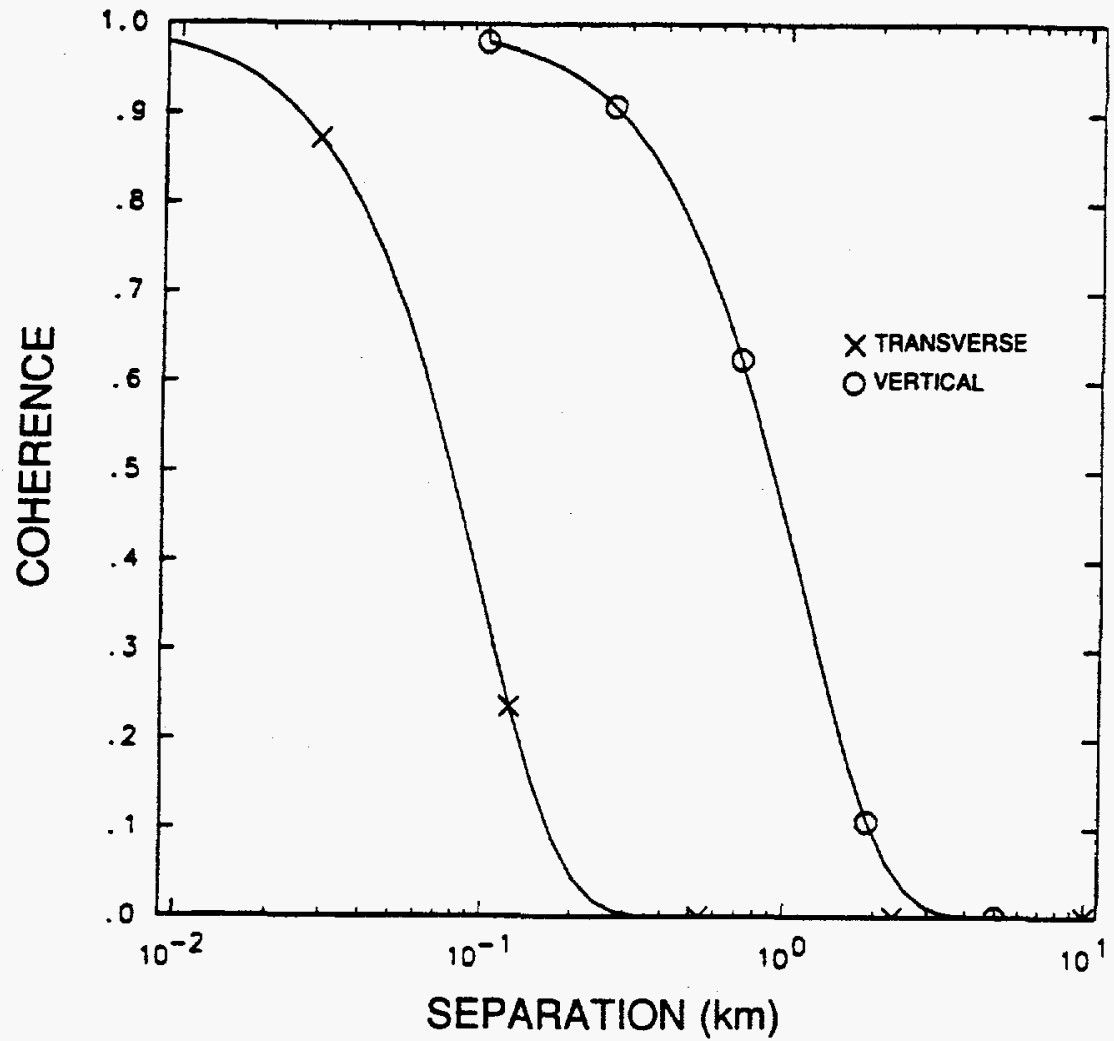

Fig. 7. Mutual coherence for receiver separations in the transverse and vertical directions for the ray shown in Figure 6 calculated using Eqs. (62) and (56).

on the specified parameters such as strength of fluctuations, type of ray path (high or low), electron density profile, orientation of the fluctuations, transmission frequency, etc.

\section{CONCLUSIONS}

The formalism developed in this paper allows us to calculate the spatial and frequency coherence of oblique, one-hop, hf paths. We thus have a method of comparing the performance of hf communication links to predictions based upon model electron density profiles and the statistics of electron density fluctuations. This comparison would most easily be made if one mode is isolated using some combination of time delay and Doppler selectivity. To predict the behavior of an actual communication link one would have to include deterministic multipath (high or low 


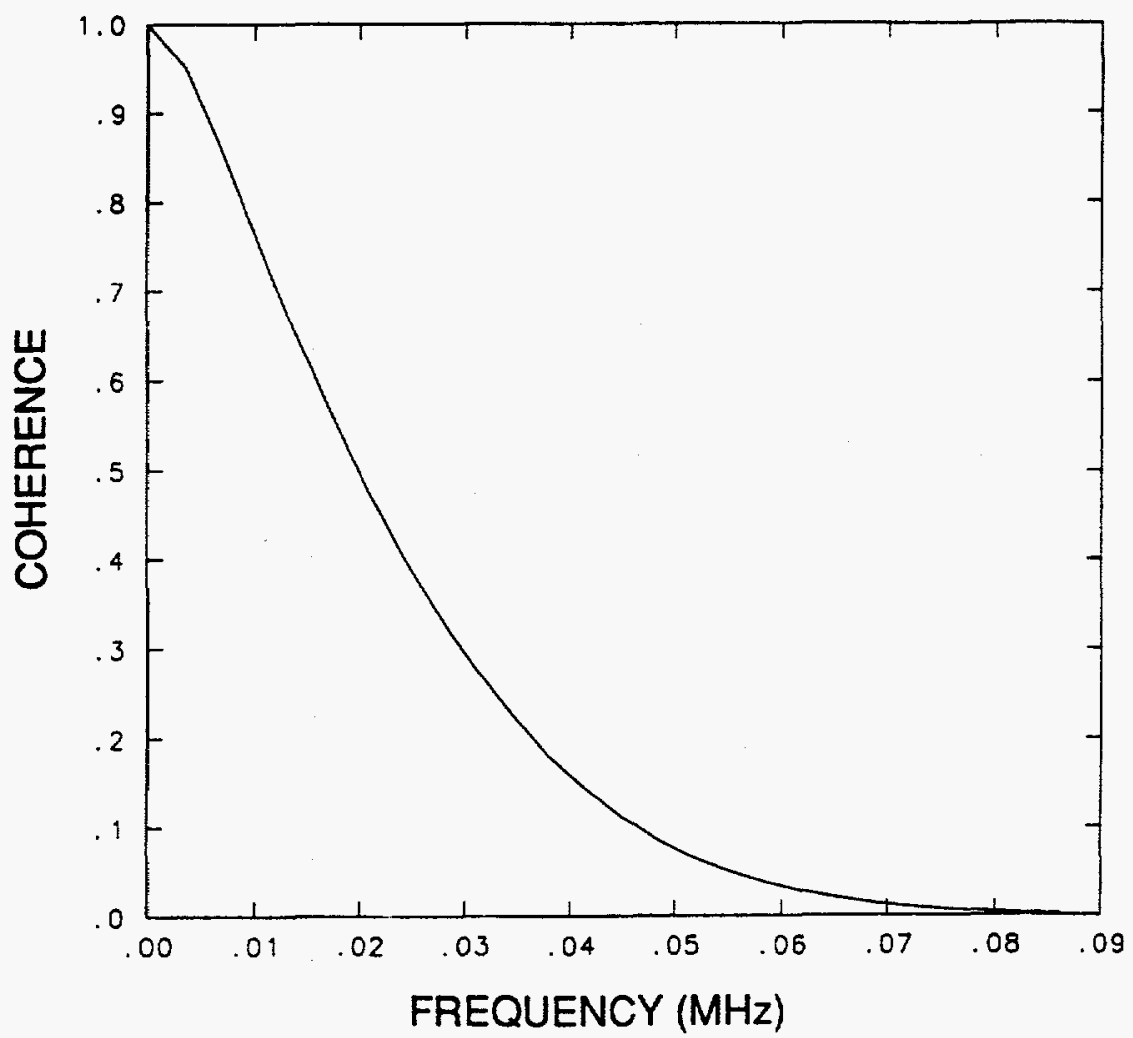

Fig. 8. Frequency coherence for the ray shown in Figure 6 calculated using Eqs. (82) and (83); the estimated half bandwidth is $20 \mathrm{kHz}$.

rays, multi-hop, etc.) and polarization splitting. Furthermore, one must be able to compensate for deterministic pulse spreading caused by dispersion in order to compare the effect of random fluctuations to this model. The difficulty of these measurements has inhibited progress in characterizing the hf ionospheric channel but it appears that given the current interest in hf communication a better understanding of this important communication link will be forthcoming.

Acknowledgment: Discussions with S. M. Flatté have been helpful and illuminating. 


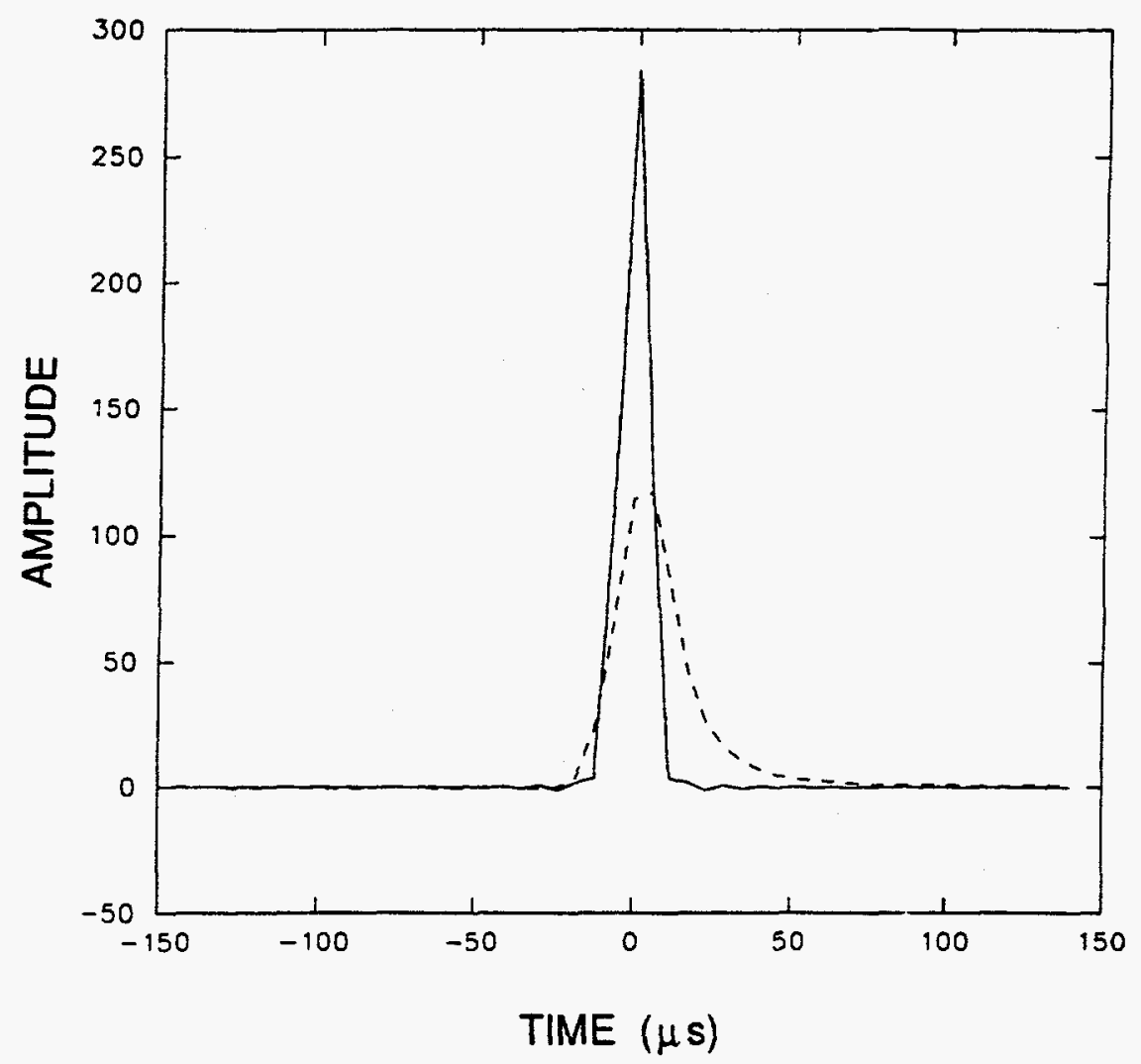

Fig. 9. Ensemble average pulse response for ray shown in Figure 6. The solid curve represents the amplitude of the transmitted pulse, a Gaussian with a full width at half-maximum of $10 \mu \mathrm{s}$. The dashed curve represents the ensemble average of the received pulse which shows a delay shift and spread relative to the nominal pulse.

\section{REFERENCES}

Basler, R. P., G. H. Price, R. T. Tsunoda, and T. L. Wong, Ionospheric distortion of hf signals, Radio Science, 23, 569-579, 1988.

Dashen, R., S. M. Flatté, and S. R. Reynolds, Path-integral treatment of acoustic mutual coherence functions for rays in a sound channel, J. Acous. Soc. Am., 77, 1716-1722, 1985.

Esswein, R., and S. M. Flatté, Calculation of the phase-structure function density from oceanic internal waves, J. Acous. Soc. Am., 70, 1387-1396, 1981. 
Feynman, R. P., and A. R. Hibbs, Quantum Mechanics and Path Integrals, McGraw-Hill, New York, 1965.

Flatté, S. M., Wave propagation through random media: contributions from ocean acoustics, Proc. IEEE, 71, 1267-1294, 1983.

Flatté, S. M., The Schrödinger equation in classical physics, Am. J. Phys., 54, 1088-1092, 1986.

Flatté, S. M., R. Dashen, W. H. Munk, K. M. Watson, and F. Zachariasen, Sound Transmission Through a Fluctuating Ocean, Cambridge University Press, New York, 1979.

Kelly, M. C., K. D. Baker, and J. C. Ulwick, Late time barium cloud striations and their possible relationship to equatorial spread F, J. Geophys. Res., 84, 1898, 1979.

Koeling, T. and R. A. Malfliet, Semi-classical approximations to heavy ion scattering based on the Feynman path-integral method, Physics Reports, 22, 181-213, 1975.

Schulman, L. S., Techniques and Applications of Path Integrals, John Wiley and Sons, New York, 1981.

Shkarofsky, I. P., Generalized turbulence space-correlation and wave-number spectrum-function pairs, Can. J. Physics, 46, 2133-2153, 1968. 